\title{
Sufficient conditions for assignability of nonuniform dichotomy spectrum of discrete time-varying linear systems
}

\author{
Artur Babiarz ${ }^{1}$, Adam Czornik ${ }^{1}$ and Stefan Siegmund ${ }^{2 *}$ (D)
}

"Correspondence:

stefan.siegmund@tu-dresden.de ${ }^{2}$ Faculty of Mathematics,

Technische Universität Dresden, 01062 Dresden, Germany

Full list of author information is

available at the end of the article

\section{Springer}

\begin{abstract}
We consider a version of the pole placement problem for tempered one-sided linear discrete-time time-varying linear systems. We prove a sufficient condition for assignability of the nonuniform dichotomy spectrum by linear feedback. The main result is that the nonuniform dichotomy spectrum is assignable if the system is completely controllable and certain lower asymptotic bound for the controllability Gramian holds.
\end{abstract}

Keywords: Discrete time-varying systems; Dichotomy; Spectrum of dichotomy; Assignability; Controllability

\section{Introduction}

The concept of exponential dichotomy is closely related to the problem of inferring the stability of a nonlinear system based on its linear approximation. This problem was first formulated by Lyapunov (see [1]), who showed that for the class of regular systems introduced by him, the stability of the linear approximation implies the local stability of the nonlinear system. In [2], Perron showed that this implication is also true for nonlinear systems, the linear approximation of which has the property that its inhomogeneous version has a bounded solution to any bounded inhomogeneity. Perron thus introduced for the first time a class of systems that we now call systems with an exponential dichotomy.

On the other hand, Maizel [3] extended the Persidskii criterion [4] to the case of conditional stability and Malkin's criterion [5] on the stability of the first approximation, and thus defined two classes of linear differential systems for which the Lyapunov problem also has a positive answer. It turned out [3] that these two classes coincide with each other and with the class of systems defined by Perron-thus, the class of exponentially dichotomous systems was characterized from three essentially different points of view.

The works of Perron and Maisel were preceded by the works of Hadamard [6] and Bohl [7], which contained essentially the same key ideas that later led to the concept of exponential dichotomy.

The class of exponentially dichotomic systems was singled out as an independent subject of research with its own problems in a series of works [8,9] by Massera and Schaeffer, who

(c) The Author(s) 2022. This article is licensed under a Creative Commons Attribution 4.0 International License, which permits use, sharing, adaptation, distribution and reproduction in any medium or format, as long as you give appropriate credit to the original author(s) and the source, provide a link to the Creative Commons licence, and indicate if changes were made. The images or other third party material in this article are included in the article's Creative Commons licence, unless indicated otherwise in a credit line to the material. If material is not included in the article's Creative Commons licence and your intended use is not permitted by statutory regulation or exceeds the permitted use, you will need to obtain permission directly from the copyright holder. To view a copy of this licence, visit http://creativecommons.org/licenses/by/4.0/. 
also coined the name "exponential dichotomy" and formulated the canonical definition of exponentially dichotomous systems.

The final formulation of fundamentals of the theory of exponentially dichotomous systems was completed thanks to the monographs of Massera and Schaeffer [10] and Daleckii and Krein [11], who summarized the earlier obtained results on exponentially dichotomous systems and outlined the development paths of this theory by setting many new problems and by formulating interesting and deep open questions.

Later, an equally important role in the development of the theory of dichotomy was played by Coppel's monograph [12], in which the results on finite-dimensional exponentially dichotomous systems obtained before 1978 were collected and strengthened.

The effectiveness of the notion of exponential dichotomy both in the study of the asymptotic behavior of solutions of nonlinear systems, the first approximation of which is exponentially dichotomous, and in its applications to dynamical systems, has caused various generalizations of this notion both within the theory of linear differential systems itself and beyond-in the theory of evolution operators and the theory of linear extensions of dynamical systems. One of them is the concept of nonuniform exponential dichotomy, which is defined in the literature in many nonequivalent ways (see [13-16]).

The concept of the dichotomy is closely related to the concept of the dichotomy spectrum, introduced by Sacker and Sell in [17]. In [17], the authors developed the Sacker-Sell spectral theory, which is now also called dichotomy spectrum for nonautonomous differential equations [18]. Nowadays, the dichotomy spectrum is an important tool in the qualitative theory of dynamical systems. This is due to the following reasons. The dichotomy spectrum, together with the associated spectral manifolds, completely describes the dynamical skeleton of a linear system. This spectrum depicts uniform exponential stability as follows: if the dichotomy spectrum lies left of zero, then the uniform exponential stability of nonlinearly perturbed systems is guaranteed [19]. More generally, this concept may be used to discuss the existence and the smoothness of invariant manifolds for nonautonomous differential equations, to obtain a version of the Grobman-Hartman theorem for nonautonomous systems (in this context, the hyperbolicity is formulated as zero does not belong to the dichotomy spectrum) [20], to characterize the existence of center manifolds [21] and in the theory of the Lyapunov regularity [22]. Using a resonance condition of the dichotomy spectrum to study the normal forms of nonautonomous system, in [18], a finite order normal form was obtained, and in [23], analytic normal forms of a class of analytic nonautonomous differential systems were presented. Finally, information on the fine structure of the dichotomy spectrum allows classifying various types of nonautonomous bifurcations [24]. In this paper, we consider nonuniform dichotomy spectrum as it was defined in [25] (see also [26]).

In control theory, one of the most effective methods of designing control systems for stationary systems is the pole placement method (see [27]). The most important result here is the fact that the controllability of a linear time-invariant system is equivalent to the fact that for each set of complex numbers whose cardinality is equal to the size of the state vector and is symmetric about the real axis, there exists a stationary feedback such that the poles of the closed-loop system form this set [28].

The generalization of this methodology to systems with time-varying coefficients encounters two main difficulties: there are many nonequivalent controllability concepts, and for systems with variable coefficients, there are many numerical characteristics that play, 
to a certain extent, the role of the poles of stationary systems (e.g., the Lyapunov, Bohl and Perron exponents or dichotomy spectra). The methods of assignability of the so-called Lyapunov invariants through linear feedback for continuous systems are fully described in the monographs of Makarov and Popova [29], and for discrete systems, the assignability of the Lyapunov exponents is discussed in the series of papers [30, 31], and [32]. The problem of assignability of the spectrum of uniform exponential dichotomy was discussed in the works [33, 34], and [35], where it was shown that both for discrete and continuous systems with bounded time-varying coefficients, considered both on the half-line and on the whole line, the spectrum of uniform exponential dichotomy is assignable if and only if the system is uniformly completely controllable.

Although a uniform dichotomy is a common phenomenon (see [10]), it may not be satisfied in many important situations, for example, when the linear equation is a result of linearization of certain nonlinear dynamics [36]. Moreover, as it was shown in this paper, analysis of such dynamics can be successfully performed using the weaker concept of nonuniform dichotomy. The corresponding spectrum of the nonuniform dichotomy is a subset of the spectrum of the uniform dichotomy, but it contains all the Lyapunov exponents as subsets, and therefore, on its basis, it is possible to infer exponential stability. For this reason, among other things, in control systems, as the aim of control can be considered, placement of the spectrum of nonuniform dichotomies in a given position, in particular by selecting the position as a set of points, leads to the task of locating Lyapunov exponents.

In this paper, we investigate the problem of the relationship between the assignability of the nonuniform dichotomy spectrum and complete controllability for discrete linear systems with not necessarily bounded time-varying coefficients.

In all previous works on the assignability of the Lyapunov exponents ([29-31], and [32]) and the dichotomy spectrum $([33,34]$, and [35]), there is an assumption that the coefficients are bounded, and this assumption plays an important role there. In the present work, it was possible to significantly weaken this assumption and obtain results on the assignability of the spectrum, assuming that the coefficients are tempered. Related to the concept of a tempered sequence is the concept of tempered exponential dichotomy. In [37], the authors characterize the concept of a tempered exponential dichotomy on a Banach space in terms of an admissibility property. Additionally, they show a new proof of the robustness property of the notion of a tempered exponential dichotomy under sufficiently small linear perturbations for continuous-time dynamical systems. For discrete-time dynamical systems, the characterization of tempered exponential dichotomy on a Banach space is presented in [38]. As a result, the authors show that the concept of an exponential dichotomy under sufficiently small parameterized perturbations perseveres and that their stable and unstable spaces are as regular as the perturbation.

For the consideration of the systems with not necessarily bounded coefficients, it was compulsory to create new methods that allowed developing the key for the main result of Theorem 12 about transforming the system through linear feedback to an upper triangular form with predetermined elements on the main diagonal. The diagonal significance of nonuniform dichotomy, i.e., the property that the spectrum of an upper triangular system is the union of spectra of one dimensional systems corresponding to the elements of the main diagonal, shown in Theorems 6 and 7, also plays an important role in proving the main result and is interesting for the theory of dichotomy itself. For the purpose of this 
result, we adapted to discrete systems the idea of the linking operator proposed in [39] for continuous systems. Finally, it should be noted that as shown in [34] and [35], the assignability of the spectrum of a uniform dichotomy requires the assumption of one of the strongest types of controllability, i.e., uniform complete controllability, while in our work, we consider a significantly weaker assumption about complete controllability.

The paper is organized as follows. In the next section, we present the basic definition, formulation of the problem, and the main result. In the third section, we discuss properties of nonuniform dichotomy for upper triangular systems. The fourth section is devoted to properties of complete controllability. The proof of the main results is presented in the fifth section. Section six contains an example. We formulate conclusions in the last section.

Denote by $\mathbb{N}$ the set of natural numbers. We denote the $s$-dimensional Euclidean space with Euclidean norm $\|\cdot\|$ and the set of matrices of size $s$ by $t$ with real entries by $\mathbb{R}^{s}$ and $\mathbb{R}^{s \times t}$, respectively. For a matrix $X \in \mathbb{R}^{s \times t},\|A\|$ denotes the operator norm generated by the Euclidean norm. $\mathrm{GL}_{s}(\mathbb{R})$ is the subset of $\mathbb{R}^{s \times s}$ consisting of invertible matrices. If $X, Y \in \mathbb{R}^{s \times s}$ are symmetric, then $X \geq Y,(X>Y)$ means that the matrix $X-Y$ is nonnegative definite (positive definite). For a sequence $A: \mathbb{N} \rightarrow \mathrm{GL}_{s}(\mathbb{R}), A=(A(k))_{k \in \mathbb{N}}$, we denote by $A^{-1}$ the sequence $\left(A(k)^{-1}\right)_{k \in \mathbb{N}}$, which we will also write as $\left(A^{-1}(k)\right)_{k \in \mathbb{N}}$. The identity matrix of size $s$ by $s$ is denoted by $I_{s}$. The set of all sequences $X: \mathbb{N} \rightarrow \mathbb{R}^{s \times t}, X=(X(k))_{k \in \mathbb{N}}$ such that

$$
\limsup _{k \rightarrow \infty} \frac{1}{k} \ln \|X(k)\| \leq 0
$$

is denoted by $\mathcal{L}^{\operatorname{tem}}\left(\mathbb{N}, \mathbb{R}^{s \times t}\right)$, and its elements are called tempered sequences.

\section{Preliminaries and statements of main results}

For a sequence $A: \mathbb{N} \rightarrow \mathrm{GL}_{s}(\mathbb{R}), A=(A(k))_{k \in \mathbb{N}}$, let $\Phi_{A}(\cdot, \cdot)$ denote the evolution operator generated by $A$, i.e.,

$$
\Phi_{A}(k, l):= \begin{cases}A(k-1) \cdots A(l), & \text { if } k>l, \\ \text { id, } & \text { if } k=l, \\ \Phi_{A}^{-1}(l, k), & \text { if } k<l .\end{cases}
$$

Consider a discrete time-varying linear system

$$
x(k+1)=A(k) x(k), \quad k \in \mathbb{N},
$$

where $A:=(A(k))_{k \in \mathbb{N}}, A(k) \in \mathrm{GL}_{s}(\mathbb{R})$. If $\left(x_{A}\left(k, k_{0}, x_{0}\right)\right)_{k \in \mathbb{N}}$ denotes the solution of (1), satisfying the condition $x_{A}\left(k_{0}, k_{0}, x_{0}\right)=x_{0}$, then

$$
x_{A}\left(k, k_{0}, x_{0}\right)=\Phi_{A}\left(k, k_{0}\right) x_{0}, \quad k \in \mathbb{N} .
$$

A matrix $\widetilde{P} \in \mathbb{R}^{s \times s}$ is called projector if $\widetilde{P}^{2}=\widetilde{P}$. An invariant projector of (1) is defined to be a function $P: \mathbb{N} \rightarrow \mathbb{R}^{s \times s}$ of projections $P(k), k \in \mathbb{N}$, such that

$$
P(k) \Phi_{A}(k, l)=\Phi_{A}(k, l) P(l), \quad k, l \in \mathbb{N} .
$$

In this paper, we consider the following definition of nonuniform exponential dichotomy (NED) and nonuniform exponential dichotomy spectrum (NEDS) introduced in [25]. 
Definition 1 (Nonuniform exponential dichotomy) We say that (1) admits a NED if there exists an invariant projector $(P(m))_{m \in \mathbb{N}}$ of (1), a constant $\alpha>0$, and for each $\varepsilon>0$ a constant $D(\varepsilon)>0$ such that for $k, l \in \mathbb{N}$

$$
\begin{aligned}
& \left\|\Phi_{A}(k, l) P(l)\right\| \leq D(\varepsilon) e^{-\alpha(k-l)+\varepsilon l}, \quad k \geq l, \\
& \left\|\Phi_{A}(k, l)\left(I_{s}-P(l)\right)\right\| \leq D(\varepsilon) e^{-\alpha(l-k)+\varepsilon l}, \quad k \leq l .
\end{aligned}
$$

Definition 2 (Nonuniform exponential dichotomy spectrum) The nonuniform exponential dichotomy spectrum (NEDS) of (1) is defined by

$$
\Sigma(A):=\left\{\gamma \in \mathbb{R}: x(k+1)=e^{-\gamma} A(k) x(k) \text { has no NED }\right\} .
$$

The structure of the NEDS is given by the following theorem from [25].

Theorem 1 (Spectral theorem) For system (1), either $\Sigma(A)=\emptyset, \Sigma(A)=\mathbb{R}$, or

$$
\Sigma(A)=I_{1} \cup\left[a_{2}, b_{2}\right] \cup \cdots \cup\left[a_{p-1}, b_{p-1}\right] \cup I_{p},
$$

where $I_{1}=\left[a_{1}, b_{1}\right]$ or $I_{1}=\left(-\infty, b_{1}\right]$, and $I_{p}=\left[a_{p}, b_{p}\right]$ or $I_{p}=\left[a_{p},+\infty\right)$, for some numbers $a_{1} \leq b_{1}<a_{2} \leq b_{2}<\cdots<a_{p} \leq b_{p}$ and $p \leq s$.

Consider now a discrete linear time-varying system with control

$$
x(k+1)=A(k) x(k)+B(k) u(k),
$$

where $A=(A(k))_{k \in \mathbb{N}}, A(k) \in \mathrm{GL}_{s}(\mathbb{R}), B: \mathbb{N} \rightarrow \mathbb{R}^{s \times t}, B=(B(k))_{k \in \mathbb{N}}$, and the control sequence $u=(u(k))_{k \in \mathbb{N}}$ is $t$-dimensional.

The (forward) solution of (5) corresponding to the control $u$ and initial condition $x\left(k_{0}\right)=$ $x_{0}$, where $k_{0} \in \mathbb{N}$ and $x_{0} \in \mathbb{R}^{s}$, is denoted by

$$
\left(x\left(k, k_{0}, x_{0}, u\right)\right)_{k \geq k_{0}}
$$

and is given by the following formula

$$
x\left(k, k_{0}, x_{0}, u\right)=\Phi_{A}\left(k, k_{0}\right) x_{0}+\sum_{j=k_{0}}^{k-1} \Phi_{A}(k, j+1) B(j) u(j), \quad k \geq k_{0} .
$$

If we have a sequence $U: \mathbb{N} \rightarrow \mathbb{R}^{t \times s}, U=(U(k))_{k \in \mathbb{N}}$, then we may define a so-called feedback control $u=(u(k))_{k \in \mathbb{N}}$ by

$$
u(k)=U(k) x(k), \quad k \in \mathbb{N} .
$$

With this control, system (5) takes the following closed-loop form

$$
x(k+1)=(A(k)+B(k) U(k)) x(k) .
$$

Our main result will be formulated under the assumption that $A, A^{-1} \in \mathcal{L}^{\text {tem }}\left(\mathbb{N}, \mathbb{R}^{s \times s}\right)$ and $B \in \mathcal{L}^{\text {tem }}\left(\mathbb{N}, \mathbb{R}^{s \times t}\right)$; therefore the following class of feedback is important. 
Definition 3 (Admissible feedback) Suppose that $A, A^{-1} \in \mathcal{L}^{\text {tem }}\left(\mathbb{N}, \mathbb{R}^{s \times s}\right), B \in \mathcal{L}^{\text {tem }}(\mathbb{N}$, $\left.\mathbb{R}^{s \times t}\right)$. A sequence $U: \mathbb{N} \rightarrow \mathbb{R}^{t \times s}, U=(U(k))_{k \in \mathbb{N}}$, which satisfies

$$
\limsup _{n \rightarrow \infty} \frac{1}{n} \ln \|U(n)\|=0,
$$

is called an admissible feedback for (5) if $A+B U:=(A(k)+B(k) U(k))_{k \in \mathbb{N}}$ is such that $A(k)+$ $B(k) U(k) \in \mathrm{GL}_{s}(\mathbb{R})$ for all $k \in \mathbb{N}$ and $A+B U,(A+B U)^{-1} \in \mathcal{L}^{\text {tem }}\left(\mathbb{N}, \mathbb{R}^{s \times s}\right)$.

Remark 1 (Tempered sequences are not exponentially growing) For a tempered sequence $X=(X(k))_{k \in \mathbb{N}} \in \mathcal{L}^{\text {tem }}\left(\mathbb{N}, \mathbb{R}^{s \times t}\right)$, there exists for all $\varepsilon>0$ a $D(\varepsilon)>0$ such that

$$
\|X(k)\| \leq D(\varepsilon) e^{\varepsilon k}, \quad k \in \mathbb{N}
$$

The next definition contains the precise statement of the main objective of this paper.

Definition 4 (Assignability of spectrum) The NEDS of (7) is called assignable if for arbitrary $1 \leq p \leq s$ and an arbitrary set $\bar{\Sigma}=\emptyset, \mathbb{R}$ or of the form (4), there exists an admissible feedback $U$ such that $\Sigma(A+B U)=\bar{\Sigma}$.

The main result of the paper, which contains sufficient conditions for assignability of NEDS, is formulated in terms of complete controllability and the controllability Gramian. The formal definitions are as follows.

Definition 5 (Complete controllability) System (5) is called $K$-completely controllable $(K \in \mathbb{N})$ if for all $\left(k_{0}, \xi\right) \in \mathbb{N} \times \mathbb{R}^{s}$, there exists a control sequence $u(\ell), \ell=k_{0}, k_{0}+1, \ldots, k_{0}+$ $K-1$, such that $x\left(k_{0}+K, k_{0}, 0, u\right)=\xi$. System (5) is called completely controllable if there exists a $K \in \mathbb{N}$ such that system (5) is $K$-completely controllable.

In the investigation of controllability, a crucial role is played by the following Kalman controllability matrix

$$
W_{A, B}(k, l)=\sum_{j=k}^{l-1} \Phi_{A}(k, j+1) B(j) B^{T}(j) \Phi_{A}^{T}(k, j+1), \quad k<l .
$$

The next theorem (see $[40,41])$ gives, in terms of the Kalman controllability matrix, necessary and sufficient conditions for complete controllability.

Theorem 2 (Characterization of complete controllability) System (5) is completely controllable if and only if there exists a natural number $K$ such that

$$
W_{A, B}\left(k_{0}, k_{0}+K\right)>0
$$

for all $k_{0} \in \mathbb{N}$.

For $k, l \in \mathbb{N}$ and $k<l$, denote by $\alpha_{A, B}(k, l)$ the smallest eigenvalue of $W_{A, B}(k, l)$.

The next theorem is the main result of the paper. 
Theorem 3 (Assignability theorem) Suppose that $A, A^{-1} \in \mathcal{L}^{\text {tem }}\left(\mathbb{N}, \mathbb{R}^{s \times s}\right)$ and $B \in \mathcal{L}^{\text {tem }}(\mathbb{N}$, $\mathbb{R}^{s \times t}$ ), system (5) is $K$-completely controllable and

$$
\liminf _{k \rightarrow \infty} \frac{1}{k} \ln \alpha_{A, B}((k-1) K, k K) \geq 0 .
$$

Then the NEDS of (5) is assignable.

\section{Upper triangular sequences and dichotomy}

In this section, we present results about the NEDS for upper triangular systems. The main role is played by the linking operator introduced by Batteli and Palmer in [39]. Our main Theorem 7 shows that the NEDS of a system with upper triangular coefficients is the union of the NEDS of the scalar systems formed from the diagonal entries.

Suppose that $A=(A(k))_{k \in \mathbb{N}}$ is an upper triangular sequence

$$
A(k)=\left[\begin{array}{cc}
\bar{A}_{1}(k) & C(k) \\
0 & \bar{A}_{2}(k)
\end{array}\right], \quad k \in \mathbb{N},
$$

where $\bar{A}_{1}: \mathbb{N} \rightarrow \mathrm{GL}_{\bar{s}}(\mathbb{R}), \bar{A}_{2}: \mathbb{N} \rightarrow \mathrm{GL}_{s-\bar{s}}(\mathbb{R}), C: \mathbb{N} \rightarrow \mathbb{R}^{\bar{s} \times(s-\bar{s})}$. Then for $k, l \in \mathbb{N}$, the matrices $\Phi_{A}(k, l)$ are upper triangular and

$$
\Phi_{A}(k, l)=\left[\begin{array}{cc}
\Phi_{\bar{A}_{1}}(k, l) & \Psi(k, l) \\
0 & \Phi_{\bar{A}_{2}}(k, l)
\end{array}\right]
$$

where

$$
\Psi(k, l)= \begin{cases}\sum_{j=l}^{k-1} \Phi_{\bar{A}_{1}}(k, j+1) C(j) \Phi_{\bar{A}_{2}}(j, l), & \text { if } k>l, \\ 0, & \text { if } k=l, \\ -\sum_{j=k}^{l-1} \Phi_{\bar{A}_{1}}(k, j+1) C(j) \Phi_{\bar{A}_{2}}(j, l), & \text { if } k<l .\end{cases}
$$

Let us denote

$$
V_{1}:=\left\{x_{0} \in \mathbb{R}^{\bar{s}}:\left(x_{\bar{A}_{1}}\left(k, 0, x_{0}\right)\right)_{k \in \mathbb{N}} \text { is bounded }\right\},
$$

$V_{2}$ any complement of $V_{1}$ in $\mathbb{R}^{\bar{s}}$ i.e. $\mathbb{R}^{\bar{s}}=V_{1} \oplus V_{2}$.

It is clear that $V_{1}$ is nonempty $\left(0 \in V_{1}\right)$ and is a linear subspace of $\mathbb{R}^{\bar{s}}$; therefore, $V_{2}$ is well-defined. For a fixed $x_{0} \in \mathbb{R}^{s-\bar{s}}$, consider the equation

$$
\bar{x}(k+1)=\bar{A}_{1}(k) \bar{x}(k)+C(k) \Phi_{\bar{A}_{2}}(k, 0) x_{0}, \quad k \in \mathbb{N},
$$

and denote its solution, satisfying $\bar{x}(0)=\bar{x}_{0} \in \mathbb{R}^{\bar{s}}$ by $\left(\bar{x}\left(k, 0, \bar{x}_{0}\right)\right)_{k \in \mathbb{N}}$. By the variation of constants, formula (see [42, pp. 83]), $\left(\bar{x}\left(k, 0, \bar{x}_{0}\right)\right)_{k \in \mathbb{N}}$ is given by

$$
\bar{x}\left(k, 0, \bar{x}_{0}\right)=\Phi_{\bar{A}_{1}}(k, 0) \bar{x}_{0}+\sum_{l=1}^{k} \Phi_{\bar{A}_{1}}(k, l) C(l-1) \Phi_{\bar{A}_{2}}(l-1,0) x_{0} .
$$


Let us denote

$$
W_{1}:=\left\{\begin{array}{c}
x_{0} \in \mathbb{R}^{s-\bar{s}}:\left(x_{\bar{A}_{2}}\left(k, 0, x_{0}\right)\right)_{k \in \mathbb{N}} \text { is bounded, and } \\
\text { there exists } \bar{x}_{0} \in \mathbb{R}^{\bar{s}} \text { such that }\left(\bar{x}\left(k, 0, \bar{x}_{0}\right)\right)_{k \in \mathbb{N}} \\
\text { is bounded }
\end{array}\right\},
$$

$W_{2}$ any complement of $W_{1}$ in $\mathbb{R}^{s-\bar{s}}$, i.e., $\mathbb{R}^{\bar{s}}=W_{1} \oplus W_{2}$.

Observe that $0 \in W_{1}$, and from the superposition principle (see [42, pp. 49]), it follows that $W_{1}$ is a subspace of $\mathbb{R}^{s-\bar{s}}$; therefore, $W_{2}$ is well-defined.

Remark 2 Suppose that $\left(\bar{x}\left(k, 0, \bar{x}_{01}\right)\right)_{k \in \mathbb{N}}$ and $\left(\bar{x}\left(k, 0, \bar{x}_{02}\right)\right)_{k \in \mathbb{N}}$ are solutions of $(12)$ (with the same $\left.x_{0}\right)$ and suppose that $\left(\bar{x}\left(k, 0, \bar{x}_{01}\right)\right)_{k \in \mathbb{N}}$ is bounded, then $\left(\bar{x}\left(k, 0, \bar{x}_{02}\right)\right)_{k \in \mathbb{N}}$ is bounded if and only if $\left(\bar{x}\left(k, 0, \bar{x}_{01}\right)-\bar{x}\left(k, 0, \bar{x}_{02}\right)\right)_{k \in \mathbb{N}}$ is a bounded solution of

$$
x(k+1)=\bar{A}_{1}(k) x(k), \quad k \in \mathbb{N},
$$

i.e., $\bar{x}_{01}-\bar{x}_{02} \in V_{1}$.

Proof Suppose that $\left(\bar{x}\left(k, 0, \bar{x}_{01}\right)\right)_{k \in \mathbb{N}}$ is a bounded solution of (12). If $\left(\bar{x}\left(k, 0, \bar{x}_{02}\right)\right)_{k \in \mathbb{N}}$ is bounded, then so is $\left(\bar{x}\left(k, 0, \bar{x}_{01}\right)-\bar{x}\left(k, 0, \bar{x}_{02}\right)\right)_{k \in \mathbb{N}}$. The fact that $\left(\bar{x}\left(k, 0, \bar{x}_{01}\right)-\bar{x}\left(k, 0, \bar{x}_{02}\right)\right)_{k \in \mathbb{N}}$ is a solution of (14) follows from the following calculation

$$
\begin{aligned}
\bar{x}(k & \left.+1,0, \bar{x}_{01}\right)-\bar{x}\left(k+1,0, \bar{x}_{02}\right) \\
& =\bar{A}(k) \bar{x}\left(k, 0, \bar{x}_{01}\right)+C(k) \Phi_{\bar{A}_{2}}(k, 0) x_{0}-\left(\bar{A}(k) \bar{x}\left(k, 0, \bar{x}_{02}\right)+C(k) \Phi_{\bar{A}_{2}}(k, 0) x_{0}\right) \\
& =\bar{A}(k)\left(\bar{x}\left(k, 0, \bar{x}_{01}\right)-\bar{x}\left(k, 0, \bar{x}_{02}\right)\right) .
\end{aligned}
$$

If $\left(\bar{x}\left(k, 0, \bar{x}_{01}\right)-\bar{x}\left(k, 0, \bar{x}_{02}\right)\right)_{k \in \mathbb{N}}$ is bounded (regardless if it is a solution of (14) or not), then the boundedness of $\left(\bar{x}\left(k, 0, \bar{x}_{01}\right)\right)_{k \in \mathbb{N}}$ implies boundedness of $\left(\bar{x}\left(k, 0, \bar{x}_{02}\right)\right)_{k \in \mathbb{N}}$.

This simple observation leads to the following nontrivial fact.

Lemma 1 For each $x_{0} \in W_{1}$, there exists exactly one $\bar{x}_{0} \in V_{2}$ such that the solution $\left(\bar{x}\left(k, 0, \bar{x}_{0}\right)\right)_{k \in \mathbb{N}}$ of $(12)$ is bounded.

Proof First, we show that for each $x_{0} \in W_{1}$, there exists $\bar{x}_{0} \in V_{2}$ such that the solution $\left(\bar{x}\left(k, 0, \bar{x}_{0}\right)\right)_{k \in \mathbb{N}}$ of $(12)$ is bounded. Since $x_{0} \in W_{1}$, there exists $\widetilde{x}_{0} \in \mathbb{R}^{\bar{s}}$ such that $\left(\bar{x}\left(k, 0, \widetilde{x}_{0}\right)\right)_{k \in \mathbb{N}}$ is bounded. Let us decompose

$$
\tilde{x}_{0}=\bar{x}_{01}+\bar{x}_{02}
$$

with $\bar{x}_{01} \in V_{1}$ and $\bar{x}_{02} \in V_{2}$. Then $\left(\bar{x}\left(k, 0, \widetilde{x}_{0}\right)-\bar{x}\left(k, 0, \bar{x}_{02}\right)\right)_{k \in \mathbb{N}}$ is solution of (14), and it is bounded since $\widetilde{x}_{0}-\bar{x}_{02}=\bar{x}_{01} \in V_{1}$. By Remark 2, we conclude that $\left(\bar{x}\left(k, 0, \bar{x}_{02}\right)\right)_{k \in \mathbb{N}}$ is a bounded solution of (12).

Suppose that for a certain $x_{0} \in W_{1}$, we have two bounded solutions $\left(\bar{x}\left(k, 0, \bar{x}_{01}\right)\right)_{k \in \mathbb{N}}$ and $\left(\bar{x}\left(k, 0, \bar{x}_{02}\right)\right)_{k \in \mathbb{N}}$ of $(12)$. Then by Remark $2,\left(\bar{x}\left(k, 0, \bar{x}_{01}\right)-\bar{x}\left(k, 0, \bar{x}_{02}\right)\right)_{k \in \mathbb{N}}$ is a bounded solution of (14), and therefore, $\bar{x}_{01}-\bar{x}_{02} \in V_{1}$. Since $\bar{x}_{01}-\bar{x}_{02} \in V_{2}$, it follows that $\bar{x}_{01}=\bar{x}_{02}$. 
Using this Lemma, we may associate with system (10) a linear operator $L: W_{1} \rightarrow V_{2}$, the so-called linking operator (see [39] and [43]), by the formula $L x_{0}=\bar{x}_{0}$, where $\bar{x}_{0}$ is the unique element in $V_{2}$ such that the solution $\left(\bar{x}\left(k, 0, \bar{x}_{0}\right)\right)_{k \in \mathbb{N}}$ of $(12)$ is bounded. By Lemma 1 , this operator is well-defined, and its linearity is obvious.

Let $\bar{P}^{\bar{A}_{1}}: \mathbb{R}^{\bar{s}} \rightarrow \mathbb{R}^{\bar{s}}$ and $Q: \mathbb{R}^{s-\bar{s}} \rightarrow \mathbb{R}^{s-\bar{s}}$ be the projections onto $V_{1}$ and $W_{1}$, respectively. We consider the projection $P: \mathbb{R}^{s} \rightarrow \mathbb{R}^{s}$ given by

$$
P=\left[\begin{array}{cc}
\bar{P}^{\bar{A}_{1}} & L Q \\
0 & Q
\end{array}\right],
$$

and define $P(m): \mathbb{R}^{s} \rightarrow \mathbb{R}^{s}$ for $m \in \mathbb{N}$ by

$$
P(m)=\Phi_{A}(m, 0) P \Phi_{A}(0, m)
$$

and $\bar{P}^{\bar{A}_{1}}(m): \mathbb{R}^{\bar{s}} \rightarrow \mathbb{R}^{\bar{s}}, Q(m): \mathbb{R}^{s-\bar{s}} \rightarrow \mathbb{R}^{s-\bar{s}}$ for $m \in \mathbb{N}$ by

$$
\bar{P}^{\bar{A}_{1}}(m)=\Phi_{\bar{A}_{1}}(m, 0) \bar{P}^{\bar{A}_{1}} \Phi_{\bar{A}_{1}}(0, m), \quad Q(m)=\Phi_{\bar{A}_{2}}(m, 0) Q \Phi_{\bar{A}_{2}}(0, m) .
$$

Observe that $(P(m))_{m \in \mathbb{N}},\left(\bar{P}^{\bar{A}}(m)\right)_{m \in \mathbb{N}}$ and $(Q(m))_{m \in \mathbb{N}}$ satisfy the invariance properties

$$
\begin{aligned}
& P(k) \Phi_{A}(k, m)=\Phi_{A}(k, m) P(m), \quad m, k \in \mathbb{N}, \\
& \bar{P}^{\bar{A}_{1}}(k) \Phi_{\bar{A}_{1}}(k, m)=\Phi_{\bar{A}_{1}}(k, m) \bar{P}^{\bar{A}_{1}}(m), \quad m, k \in \mathbb{N}, \\
& Q(k) \Phi_{\bar{A}_{2}}(k, m)=\Phi_{\bar{A}_{2}}(k, m) Q(m), \quad m, k \in \mathbb{N} .
\end{aligned}
$$

The following remark follows directly from Definition 1 of nonuniform exponential dichotomy.

Remark 3 (Alternative characterizations of NED) The following three statements are equivalent:

(i) System (1) admits a NED.

(ii) There exists an invariant projector $(P(m))_{m \in \mathbb{N}}$, a constant $\alpha>0$, and for each $\varepsilon>0$ a constant $D(\varepsilon)>0$ such that for $k, l \in \mathbb{N}$ and $x \in \mathbb{R}^{s}$

$$
\begin{aligned}
& \left\|\Phi_{A}(l, k) P(k) x\right\| \leq D(\varepsilon) e^{-\alpha(l-k)+\varepsilon k}\|x\|, \quad l \geq k, \\
& \left\|\Phi_{A}(l, k)\left(I_{s}-P(k)\right) x\right\| \leq D(\varepsilon) e^{-\alpha(k-l)+\varepsilon k}\|x\|, \quad l \leq k .
\end{aligned}
$$

(iii) There exists a projection $P$, a constant $\alpha>0$, and for each $\varepsilon>0$ a constant $D(\varepsilon)>0$ such that for $k, l \in \mathbb{N}$

$$
\begin{aligned}
& \left\|\Phi_{A}(l, 0) P \Phi_{A}^{-1}(k, 0)\right\| \leq D(\varepsilon) e^{-\alpha(l-k)+\varepsilon k}, \quad l \geq k, \\
& \left\|\Phi_{A}(l, 0)\left(I_{s}-P\right) \Phi_{A}^{-1}(k, 0)\right\| \leq D(\varepsilon) e^{-\alpha(k-l)+\varepsilon k}, \quad l \leq k .
\end{aligned}
$$

Remark 4 It is clear that if the inequalities (16) and (17) hold for all $\varepsilon \in\left(0, \varepsilon^{\prime}\right)$ for a certain $\varepsilon^{\prime}>0$, then they hold for all $\varepsilon>0$. Therefore, to show that a system has a NED it is enough to show that (16) and (17) hold for all sufficiently small $\varepsilon>0$. 
Lemma 2 (Uniqueness of the image of the projector) Suppose that system (1) admits a $N E D$. Then for each $k \in \mathbb{N}$,

$$
\operatorname{Im} P(k)=\left\{v \in \mathbb{R}^{s}: \sup _{m \geq k}\left\|\Phi_{A}(m, k) v\right\|<\infty\right\},
$$

and

$$
\operatorname{Im} P=\operatorname{Im} P(0)=\left\{v \in \mathbb{R}^{s}: \sup _{m \geq 0}\left\|\Phi_{A}(m, 0) v\right\|<\infty\right\}
$$

where $P(k)$ are any projections from Definition 1 , and $P$ is any projection from Remark 3(iii). In particular, the images of the projections $P(k), k \in \mathbb{N}$, and $P$, satisfying Definition 1 and Remark 3(iii), respectively, are unique.

Proof The equality

$$
\operatorname{Im} P(k)=\left\{v \in \mathbb{R}^{s}: \sup _{m \geq k}\left\|\Phi_{A}(m, k) v\right\|<\infty\right\}, \quad k \in \mathbb{N},
$$

is proved in [26, Proposition 1]. If $P$ is any projection from Remark 3(iii), it is easy to show that the projections

$$
P(k)=\Phi_{A}(k, 0) P \Phi_{A}^{-1}(k, 0), \quad k \in \mathbb{N},
$$

satisfy Definition 1, and therefore,

$$
\operatorname{Im} P(0)=\operatorname{Im} P .
$$

Theorem 4 (NED for blocks of the upper triangular system) Suppose that (10) admits a $N E D$ on $\mathbb{N}$ with invariant projections $(P(m))_{m \in \mathbb{N}}$ and constant $\alpha>0$. Then both systems

$$
\bar{x}(k+1)=\bar{A}_{1}(k) \bar{x}(k) \quad \text { for } k \in \mathbb{N}
$$

and

$$
y(k+1)=\bar{A}_{2}(k) y(k) \quad \text { for } k \in \mathbb{N},
$$

have a NED with constant $\alpha>0$. Moreover, the invariant projector $(P(m))_{m \in \mathbb{N}}$ of the dichotomy for (10) can be taken in the block upper triangular form

$$
P(m)=\Phi_{A}(m, 0)\left[\begin{array}{cc}
\bar{P}^{\bar{A}_{1}} & L Q \\
0 & Q
\end{array}\right] \Phi_{A}^{-1}(m, 0) .
$$

Proof Observe that for any $\bar{x} \in \mathbb{R}^{\bar{s}}$ and $m \in \mathbb{N}$, we have

$$
P(m)\left[\begin{array}{l}
\bar{x} \\
0
\end{array}\right]=\left[\begin{array}{c}
\bar{P}^{\bar{A}_{1}}(m) \bar{x} \\
0
\end{array}\right]
$$


and

$$
\left(I_{s}-P(m)\right)\left[\begin{array}{l}
\bar{x} \\
0
\end{array}\right]=\left[\begin{array}{c}
\left(I_{\bar{s}}-\bar{P}^{\bar{A}_{1}}(m)\right) \bar{x} \\
0
\end{array}\right] .
$$

As a consequence

$$
\left\|\bar{P}^{\bar{A}}(m)\right\| \leq\|P(m)\|,
$$

and for $\bar{x} \in \mathbb{R}^{\bar{s}}$ and $m \geq k$, we get for a fixed $\varepsilon>0$

$$
\begin{aligned}
\left\|\Phi_{\bar{A}}(m, k) \bar{P}^{\bar{A}}(k) \bar{x}\right\| & =\left\|\Phi_{A}(m, k)\left[\begin{array}{c}
\bar{P}^{\bar{A}_{1}}(k) \bar{x} \\
0
\end{array}\right]\right\| \\
& =\left\|\Phi_{A}(m, k) P(k)\left[\begin{array}{c}
\bar{x} \\
0
\end{array}\right]\right\| \\
& \leq D(\varepsilon) e^{-\alpha(m-k)+\varepsilon k}\left\|\left[\begin{array}{c}
\bar{x} \\
0
\end{array}\right]\right\| \\
& =D(\varepsilon) e^{-\alpha(m-k)+\varepsilon k}\|\bar{x}\|
\end{aligned}
$$

and similarly for any $\bar{x} \in \mathbb{R}^{\bar{s}}$ and $m \leq k$

$$
\begin{aligned}
\left\|\Phi_{\bar{A}}(m, k)\left(I_{\bar{s}}-\bar{P}^{\bar{A}}(m)\right) \bar{x}\right\| & =\left\|\Phi_{A}(m, k)\left[\begin{array}{c}
\left(I_{\bar{s}}-\bar{P}^{\bar{A}_{1}}(m)\right) \bar{x} \\
0
\end{array}\right]\right\| \\
& =\left\|\Phi_{A}(m, k)\left(I_{s}-P(k)\right)\left[\begin{array}{c}
\bar{x} \\
0
\end{array}\right]\right\| \\
& \leq D(\varepsilon) e^{-\alpha(k-m)+\varepsilon k}\left\|\left[\begin{array}{c}
\bar{x} \\
0
\end{array}\right]\right\| \\
& =D(\varepsilon) e^{-\alpha(k-m)+\varepsilon k}\|\bar{x}\| .
\end{aligned}
$$

Using Remark 3, this completes the proof of NED of (18). The fact that (19) has a NED can be proven by considering the system exactly as in step 2 of the proof of Theorem 1 in [39].

From the above theorem and its proof, the following remark follows.

Remark 5 (NED projector rank for upper triangular system) If system (5) with coefficient of the upper triangular form (10) admits a NED, then $W_{1}=\operatorname{Im} Q, V_{2}=\left(\bar{P}^{\bar{A}}\right)^{\perp}$, and therefore,

$$
\operatorname{rank} P(0)=\operatorname{rank} \bar{P}^{\bar{A}_{1}}+\operatorname{rank} Q
$$

Remark 6 (NED projector for the upper triangular systems) Suppose that system (5) with the coefficient of the upper triangular form (10) admits a NED. By Theorem 4, the projector can be taken in the form (20). Using the definitions of $P(m), \bar{P}^{\bar{A}_{1}}(m), Q(m)$, and (11), it 
follows that

$$
P(m)=\left[\begin{array}{cc}
\bar{P}^{\bar{A}_{1}}(m) & R(m) \\
0 & Q(m)
\end{array}\right],
$$

where

$$
R(0)=L Q(0)
$$

and

$$
\begin{aligned}
R(m+1)= & -\bar{A}_{1}(m) \bar{P}^{\bar{A}_{1}}(m) \bar{A}_{1}^{-1}(m) C(m) \bar{A}_{2}^{-1}(m)+ \\
& +\bar{A}_{1}(m) R(m) \bar{A}_{2}^{-1}(m)+C(m) Q(m) \bar{A}_{2}^{-1}(m) .
\end{aligned}
$$

In fact, we have

$$
\begin{aligned}
P(m+1)= & {\left[\begin{array}{cc}
\bar{P}^{\bar{A}_{1}}(m+1) & R(m+1) \\
0 & Q(m+1)
\end{array}\right]=A(m) P(m) A^{-1}(m) } \\
= & {\left[\begin{array}{cc}
\bar{A}_{1}(m) & C(m) \\
0 & \bar{A}_{2}(m)
\end{array}\right]\left[\begin{array}{cc}
\bar{P}^{\bar{A}_{1}}(m) & R(m) \\
0 & Q(m)
\end{array}\right]\left[\begin{array}{cc}
\bar{A}_{1}^{-1}(m) & -\bar{A}_{1}^{-1}(m) C(m) \bar{A}_{2}^{-1}(m) \\
0 & \bar{A}_{2}^{-1}(m)
\end{array}\right] } \\
= & {\left[\begin{array}{cc}
\bar{A}_{1}(m) \bar{P}^{\bar{A}_{1}}(m) & \bar{A}_{1}(m) R(m)+C(m) Q(m) \\
0 & \bar{A}_{2}(m) Q(m)
\end{array}\right] } \\
& \times\left[\begin{array}{cc}
\bar{A}_{1}^{-1}(m) & -\bar{A}_{1}^{-1}(m) C(m) \bar{A}_{2}^{-1}(m) \\
0 & \bar{A}_{2}^{-1}(m)
\end{array}\right] \\
= & {\left[\begin{array}{cc}
\bar{A}_{1}(m) \bar{P}^{\bar{A}_{1}}(m) \bar{A}_{1}^{-1}(m) & E(m) \\
0 & \bar{A}_{2}(m) Q(m) \bar{A}_{2}^{-1}(m)
\end{array}\right], }
\end{aligned}
$$

where

$$
\begin{aligned}
E(m)= & -\bar{A}_{1}(m) \bar{P}^{\bar{A}_{1}}(m) \bar{A}_{1}^{-1}(m) C(m) \bar{A}_{2}^{-1}(m) \\
& +\bar{A}_{1}(m) R(m) \bar{A}_{2}^{-1}(m)+C(m) Q(m) \bar{A}_{2}^{-1}(m) .
\end{aligned}
$$

By induction, it can be proven that the explicit solution of (21) is given by

$$
\begin{aligned}
R(m)= & -\sum_{j=0}^{m-1} \Phi_{\bar{A}_{1}}(m, j+1) \bar{P}^{\bar{A}_{1}}(j+1) C(j)\left(I_{s-\bar{s}}-Q(j)\right) \Phi_{\bar{A}_{2}}(j, m)- \\
& -\sum_{j=m}^{\infty} \Phi_{\bar{A}_{1}}(m, j+1)\left(I_{\bar{s}}-\bar{P}^{\bar{A}_{1}}(j+1)\right) C(j) Q(j) \Phi_{\bar{A}_{2}}(j, m) .
\end{aligned}
$$

Now we will show that the opposite implication to this from Theorem 4 holds for tempered sequences $C$. 
Theorem 5 (NED for the upper triangular tempered system) Suppose that $(C(k))_{k \in \mathbb{N}} \in$ $\mathcal{L}^{\text {tem }}\left(\mathbb{N}, \mathbb{R}^{\bar{s} \times(s-\bar{s})}\right)$ and that the systems (18) and (19) admit a NED. Then also system (5) with coefficient of the upper triangular form (10) admits a NED.

Proof We show that (10) has a NED with the projector

$$
P(m)=\left[\begin{array}{cc}
\bar{P}^{\bar{A}}(m) & R(m) \\
0 & Q(m)
\end{array}\right]
$$

where $(R(m))_{k \in \mathbb{N}}$ are given by (21). Let us fix $\varepsilon>0$. First, we show that $(A(k))_{k \in \mathbb{N}}$ satisfies (16) for a certain $D(\varepsilon)>0$. Observe that

$$
\Phi_{A}(m, k) P(k)=\left[\begin{array}{cc}
\Phi_{\bar{A}}(m, k) \bar{P}^{\bar{A}}(m) & \Psi(m, k) Q(k)+\Phi_{\bar{A}}(m, k) R(k) \\
0 & \Phi_{\bar{A}_{2}}(m, k) Q(k)
\end{array}\right] .
$$

We have

$$
\begin{aligned}
\Psi(m, k) Q(k)= & \sum_{j=1}^{m-1} \Phi_{A}(m, j+1) \bar{P}^{\bar{A}}(j+1) C(j) Q(j) \Phi_{\bar{A}_{2}}(j, k) \\
& +\sum_{j=1}^{m-1} \Phi_{A}(m, j+1)\left(I_{\bar{s}}-\bar{P}^{\bar{A}}(j+1)\right) C(j) Q(j) \Phi_{\bar{A}_{2}}(j, k),
\end{aligned}
$$

and by (21), we also have

$$
\begin{aligned}
\Phi_{\bar{A}}(m, k) R(k)= & -\sum_{j=0}^{k-1} \Phi_{\bar{A}}(m, j+1) \bar{P}^{\bar{A}}(j+1) C(j)\left(I_{s-\bar{s}}-Q(j)\right) \Phi_{\bar{A}_{2}}(j, k) \\
& -\sum_{j=k}^{\infty} \Phi_{\bar{A}}(m, j+1)\left(I_{\bar{s}}-\bar{P}^{\bar{A}}(j+1)\right) C(j) Q(j) \Phi_{\bar{A}_{2}}(j, k) .
\end{aligned}
$$

Hence,

$$
\begin{aligned}
& \Psi(m, k) Q(k)+\Phi_{\bar{A}}(m, k) R(k) \\
& =\sum_{j=k}^{m-1} \Phi_{\bar{A}}(m, j+1) \bar{P}^{\bar{A}}(j+1) C(j) Q(j) \Phi_{\bar{A}_{2}}(j, k) \\
& \quad-\sum_{j=m}^{\infty} \Phi_{\bar{A}}(m, j+1)\left(I_{\bar{s}}-\bar{P}^{\bar{A}}(j+1)\right) C(j) Q(j) \Phi_{\bar{A}_{2}}(j, k) \\
& \quad-\sum_{j=0}^{k-1} \Phi_{\bar{A}}(m, j+1) \bar{P}^{\bar{A}}(j+1) C(j)\left(I_{s-\bar{s}}-Q(j)\right) \Phi_{\bar{A}_{2}}(j, k) .
\end{aligned}
$$

Let $D^{\prime}(\varepsilon)>0$ and $\alpha>0$ be such that (by Remark 4, we may assume without loss of generality that $\alpha>2 \varepsilon$ )

$$
\left\|\Phi_{\bar{A}}(m, k) \bar{P}^{\bar{A}}(k)\right\| \leq D^{\prime}(\varepsilon) e^{-\alpha(m-k)+\varepsilon k}, \quad k, m \in \mathbb{N}, m \geq k
$$




$$
\begin{aligned}
& \left\|\Phi_{\bar{A}}(m, k)\left(I_{\bar{s}}-\bar{P}^{\bar{A}}(k)\right)\right\| \leq D(\varepsilon) e^{-\alpha(k-m)+\varepsilon k}, \quad k, m \in \mathbb{N}, m \leq k, \\
& \left\|\Phi_{\bar{A}_{2}}(m, k) Q(k)\right\| \leq D^{\prime}(\varepsilon) e^{-\alpha(m-k)+\varepsilon k}, \quad k, m \in \mathbb{N}, m \geq k,
\end{aligned}
$$

and

$$
\left\|\Phi_{\bar{A}_{2}}(m, k)\left(I_{s-\bar{s}}-Q(k)\right)\right\| \leq D(\varepsilon) e^{-\alpha(k-m)+\varepsilon k}, \quad k, m \in \mathbb{N}, m \leq k .
$$

Then for $m \geq k$, we have

$$
\begin{aligned}
& \left\|\Psi(m, k) Q(k)+\Phi_{\bar{A}}(m, k) R(k)\right\| \\
& \leq\left(D^{\prime}(\varepsilon)\right)^{2} \sum_{j=k}^{m-1} e^{-\alpha(m-j-1)+\varepsilon(j+1)}\|C(j)\| e^{-\alpha(j-k)+\varepsilon k} \\
& \quad+\left(D^{\prime}(\varepsilon)\right)^{2} \sum_{j=m}^{\infty} e^{-\alpha(j+1-m)+\varepsilon(j+1)}\|C(j)\| e^{-\alpha(j-k)+\varepsilon k} \\
& \quad+\left(D^{\prime}(\varepsilon)\right)^{2} \sum_{j=0}^{k-1} e^{-\alpha(m-j-1)+\varepsilon(j+1)}\|C(j)\| e^{-\alpha(k-j)+\varepsilon k} .
\end{aligned}
$$

Since $(C(k))_{k \in \mathbb{N}}$ is tempered; therefore, there exists $d>0$ such that $\|C(k)\| \leq d e^{\varepsilon k}, k \in \mathbb{N}$. We get

$$
\begin{aligned}
&\left\|\Psi(m, k) Q(k)+\Phi_{\bar{A}}(m, k) R(k)\right\| \\
& \leq d\left(D^{\prime}(\varepsilon)\right)^{2} \sum_{j=k}^{m-1} e^{-\alpha(m-j-1)+\varepsilon(j+1)} e^{\varepsilon j} e^{-\alpha(j-k)+\varepsilon k} \\
&+d\left(D^{\prime}(\varepsilon)\right)^{2} \sum_{j=m}^{\infty} e^{-\alpha(j+1-m)+\varepsilon j} e^{\varepsilon(j+1)} e^{-\alpha(j-k)+\varepsilon k} \\
&+d\left(D^{\prime}(\varepsilon)\right)^{2} \sum_{j=0}^{k-1} e^{-\alpha(m-j-1)+\varepsilon(j+1)} e^{\varepsilon j} e^{-\alpha(k-j)+\varepsilon k} \\
&= d\left(D^{\prime}(\varepsilon)\right)^{2} e^{-\alpha m+\alpha+\alpha k+\varepsilon k+\varepsilon} \frac{e^{2 m \varepsilon}-e^{2 k \varepsilon}}{e^{2 \varepsilon}-1} \\
&+d\left(D^{\prime}(\varepsilon)\right)^{2} e^{\alpha m+\alpha k+\varepsilon k-\alpha+\varepsilon} \frac{e^{2 m \varepsilon-2 m \alpha}}{1-e^{2(\varepsilon-\alpha)}} \\
&+d\left(D^{\prime}(\varepsilon)\right)^{2} e^{-\alpha m-\alpha k+\varepsilon k+\alpha+\varepsilon} \frac{e^{2 k \alpha+2 k \varepsilon}-1}{e^{2 \alpha+2 \varepsilon}-1} \\
&= d\left(D^{\prime}(\varepsilon)\right)^{2} \frac{e^{-\alpha m+\alpha+\alpha k+\varepsilon k+\varepsilon+2 m \varepsilon}-e^{-\alpha m+\alpha+\alpha k+3 \varepsilon k+\varepsilon}}{e^{2 \varepsilon}-1} \\
&+d\left(D^{\prime}(\varepsilon)\right)^{2} \frac{e^{-\alpha m+\alpha k+\varepsilon k-\alpha+2 m \varepsilon+\varepsilon}}{1-e^{2(\varepsilon-\alpha)}} \\
&+d\left(D^{\prime}(\varepsilon)\right)^{2} \frac{e^{-\alpha m+\alpha k+\varepsilon k+\alpha+\varepsilon+2 k \varepsilon}-e^{-\alpha m-\alpha k+\varepsilon k+\alpha+\varepsilon}}{e^{2 \alpha+2 \varepsilon}-1} .
\end{aligned}
$$


Observe that for all $\varepsilon>0$, we have $e^{2 \varepsilon}-1<e^{2 \alpha+2 \varepsilon}-1$, and for sufficiently small $\varepsilon>0$, we have $e^{2 \varepsilon}-1<1-e^{2(\varepsilon-\alpha)}$, and therefore,

$$
\begin{aligned}
\| \Psi & (m, k) Q(k)+\Phi_{\bar{A}}(m, k) R(k) \| \\
\leq & \frac{d\left(D^{\prime}(\varepsilon)\right)^{2}}{e^{2 \varepsilon}-1}\left(e^{-\alpha m+\alpha+\alpha k+\varepsilon k+\varepsilon+2 m \varepsilon}-e^{-\alpha m+\alpha+\alpha k+3 \varepsilon k+\varepsilon}\right. \\
& \left.+e^{-\alpha m+\alpha k+\varepsilon k-\alpha+2 m \varepsilon+\varepsilon}+e^{-\alpha m+\alpha k+\alpha+\varepsilon+3 k \varepsilon}-e^{-\alpha m-\alpha k+\varepsilon k+\alpha+\varepsilon}\right) \\
= & \frac{d\left(D^{\prime}(\varepsilon)\right)^{2}}{e^{2 \varepsilon}-1}\left(e^{-\alpha m+\alpha+\alpha k+\varepsilon k+\varepsilon+2 m \varepsilon}+e^{-\alpha m+\alpha k+\varepsilon k-\alpha+2 m \varepsilon+\varepsilon}\right. \\
& \left.-e^{-\alpha m-\alpha k+\varepsilon k+\alpha+\varepsilon}\right) \\
= & \frac{d\left(D^{\prime}(\varepsilon)\right)^{2} e^{\alpha+\varepsilon}}{e^{2 \varepsilon}-1} e^{-\alpha(m-k)+\varepsilon k}\left(e^{2 m \varepsilon}+e^{-2 \alpha+2 m \varepsilon}-e^{-2 \alpha k}\right) \\
= & \frac{d\left(D^{\prime}(\varepsilon)\right)^{2} e^{\alpha+\varepsilon}}{e^{2 \varepsilon}-1} e^{-\alpha(m-k)+\varepsilon k+2 m \varepsilon}\left(1+e^{-2 \alpha}-e^{-2 \alpha k-2 m \varepsilon}\right) \\
= & \frac{d\left(D^{\prime}(\varepsilon)\right)^{2} e^{\alpha+\varepsilon}}{e^{2 \varepsilon}-1} e^{(2 \varepsilon-\alpha)(m-k)+3 \varepsilon k}\left(1+e^{-2 \alpha}-e^{-2 \alpha k-2 m \varepsilon}\right) \\
\leq & \frac{2 d\left(D^{\prime}(\varepsilon)\right)^{2} e^{\alpha+\varepsilon}}{e^{2 \varepsilon}-1} e^{(2 \varepsilon-\alpha)(m-k)+3 \varepsilon k} \\
= & D(\varepsilon) e^{\alpha^{\prime}(m-k)+3 \varepsilon k},
\end{aligned}
$$

where

$$
D(\varepsilon)=\frac{2 d\left(D^{\prime}(\varepsilon)\right)^{2} e^{\alpha+\varepsilon}}{e^{2 \varepsilon}-1}, \quad \alpha^{\prime}=-2 \varepsilon+\alpha .
$$

In the last step, we used the fact that

$$
1+e^{-2 \alpha}-e^{-2 \alpha k-2 m \varepsilon}<2 .
$$

Summarizing, we have proved that for each $\varepsilon^{\prime} \in(0, \alpha / 6)$, there exists $D\left(\varepsilon^{\prime}\right)>0$ such that

$$
\left\|\Psi(m, k) Q(k)+\Phi_{\bar{A}}(m, k) R(k)\right\| \leq D\left(\varepsilon^{\prime}\right) e^{\alpha^{\prime}(m-k)+\varepsilon^{\prime} k}, \quad m>k .
$$

This, together with the fact that the systems (18) and (19) admit a NED and (22), implies (16). Suppose now that $k>m$. Using the identities

$$
\begin{aligned}
\Phi_{A}(m, k)\left(I_{s}-P(k)\right) \\
\quad=\left[\begin{array}{cc}
\left(I_{\bar{s}}-\bar{P}^{\bar{A}}(m)\right) \Phi_{\bar{A}}(m, k) & \left(I_{\bar{s}}-\bar{P}^{\bar{A}}(m)\right) \Psi(m, k)-R(m) \Phi_{\bar{A}}(m, k) \\
0 & \left(I_{s-\bar{s}}-Q(k)\right) \Phi_{\bar{A}_{2}}(m, k)
\end{array}\right]
\end{aligned}
$$

and

$$
\begin{aligned}
\left(I_{\bar{s}}\right. & \left.-\bar{P}^{\bar{A}}(m)\right) \Psi(m, k)-R(m) \Phi_{\bar{A}}(m, k) \\
& =\sum_{j=k}^{\infty} \Phi_{\bar{A}}(m, j+1)\left(I_{\bar{s}}-\bar{P}^{\bar{A}}(j+1)\right) C(j) Q(j) \Phi_{\bar{A}_{2}}(j, k)
\end{aligned}
$$




$$
\begin{aligned}
& -\sum_{j=k}^{m-1} \Phi_{\bar{A}}(m, j+1)\left(I_{\bar{s}}-\bar{P}^{\bar{A}}(j+1)\right) C(j)\left(I_{s-\bar{s}}-Q(j)\right) \Phi_{\bar{A}_{2}}(j, k) \\
& +\sum_{j=0}^{m-1} \Phi_{\bar{A}}(m, j+1) \bar{P}^{\bar{A}}(j+1) C(j)\left(I_{s-\bar{s}}-Q(j)\right) \Phi_{\bar{A}_{2}}(j, k),
\end{aligned}
$$

we can show analogically, as in the case $k<m$, that (17) holds.

Now, we will consider system (1) with $A$ being in upper triangular form, i.e.,

$$
A(k)=\left[\begin{array}{ccccc}
a_{11}(k) & a_{12}(k) & a_{13}(k) & \ldots & a_{1 s}(k) \\
0 & a_{22}(k) & a_{23}(k) & \ldots & a_{2 s}(k) \\
0 & 0 & a_{33}(k) & \ldots & a_{3 s}(k) \\
\vdots & \vdots & \vdots & \ddots & \vdots \\
0 & 0 & 0 & \ldots & a_{s s}(k)
\end{array}\right] .
$$

Denote

$$
\begin{aligned}
\bar{D}(k) & =\left[\begin{array}{ccccc}
a_{11}(k) & a_{12}(k) & a_{13}(k) & \ldots & a_{1 s-1}(k) \\
0 & a_{22}(k) & a_{23}(k) & \ldots & a_{2 s-1}(k) \\
0 & 0 & a_{33}(k) & \ldots & a_{3 s-1}(k) \\
\vdots & \vdots & \vdots & \ddots & \vdots \\
0 & 0 & 0 & \ldots & a_{s-1 s-1}(k)
\end{array}\right], \\
\bar{E}(k) & =\left[\begin{array}{c}
a_{1 s}(k) \\
a_{2 s}(k) \\
a_{3 s}(k) \\
\vdots \\
a_{s-1 s}(k)
\end{array}\right] \text { and } \bar{F}(k)=a_{s s}(k) .
\end{aligned}
$$

Theorem 6 (Characterization of NED for the upper triangular tempered system) Suppose that $(A(k))_{k \in \mathbb{N}},\left(A^{-1}(k)\right)_{k \in \mathbb{N}} \in \mathcal{L}^{\text {tem }}\left(\mathbb{N}, \mathbb{R}^{s \times s}\right)$ and that $(A(k))_{k \in \mathbb{N}}$ is in the upper triangular form (23). Then (1) admits a NED if and only if every scalar equation

$$
x_{j}(k+1)=a_{j j}(k) x_{j}(k), \quad j=1, \ldots, s,
$$

admits a NED.

Proof We prove the theorem by induction over $j \in\{1, \ldots, s\}$. When $j=1$, the conclusion is obvious. When $j=2$, the conclusion follows from Theorems 4 and 5 . Suppose that the result is true when the system is $(s-1)$-dimensional. Since system (1) with $A$ in the form (23) has the form (10) with

$$
\bar{A}_{1}(k)=\bar{D}(k), \quad C(k)=\bar{E}(k) \quad \text { and } \quad \bar{A}_{2}(k)=\bar{F}(k),
$$

the conclusion of the theorem for $s$-dimensional systems follows from Theorems 4 and 5 , and the fact that if $(A(k))_{k \in \mathbb{N}}$ is tempered, then, so are $(\bar{D}(k))_{k \in \mathbb{N}},(\bar{E}(k))_{k \in \mathbb{N}}$, and $(\bar{F}(k))_{k \in \mathbb{N}}$. 
From Theorem 6, the following pivotal result follows.

Theorem 7 (NEDS for the upper triangular tempered systems) Suppose that the sequence $(A(k))_{k \in \mathbb{N}}$ in form (23) is tempered. Then

$$
\Sigma(A)=\bigcup_{j=1}^{s} \Sigma\left(a_{j j}\right),
$$

where $\Sigma\left(a_{i j}\right)$ is the NEDS of (24).

\section{Complete controllability}

This paragraph contains some considerations about complete controllability, which led to the main result formulated in Theorem 12. This theorem is a generalization of Theorem 4.6 in [30] to systems with unbounded coefficients.

The proof of the following lemma is contained in the proof of Proposition 7 in [44].

Lemma 3 (Complete controllability persists under feedback) If system (5) is $K$-completely controllable, then for any $U: \mathbb{N} \rightarrow \mathbb{R}^{t \times s}, U=(U(k))_{k \in \mathbb{N}}$, the system

$$
\bar{x}(k+1)=(A(k)+B(k) U(k)) \bar{x}(k)+B(k) \bar{u}(k)
$$

is $K$-completely controllable.

If $D=(D(k))_{k \in \mathbb{N}}$ is a sequence of invertible $s$ by $s$ matrices, then with

$$
y(k)=D(k) x(k),
$$

we get

$$
\begin{aligned}
y(k+1) & =D(k+1) x(k+1)=D(k+1)(A(k) x(k)+B(k) u(k)) \\
& =D(k+1) A(k) D^{-1}(k) y(k)+D(k+1) B(k) u(k) .
\end{aligned}
$$

In such a situation, we will say that $D$ transforms system (5) into the system

$$
y(k+1)=\bar{A}(k) y(k)+\bar{B}(k) u(k)
$$

where

$$
\bar{A}(k)=D(k+1) A(k) D^{-1}(k), \quad \bar{B}(k)=D(k+1) B(k) .
$$

In our investigation, we will use the concept of weak equivalence. In the literature, one can find various definitions of equivalence for different types of dynamical systems (e.g., see $[45-48])$.

Definition 6 (Weak equivalence) If there exists a sequence $D$ such that $D, D^{-1} \in \mathcal{L}^{\text {tem }}(\mathbb{N}$, $\mathbb{R}^{s \times s}$ ), which transforms system (5) into (27), then systems (5) and (27) are called weakly equivalent. 
Lemma 4 (Weak equivalence preserves complete controllability) Suppose that (5) is completely controllable and a transformation $D=(D(k))_{k \in \mathbb{N}}, D, D^{-1} \in \mathcal{L}^{\text {tem }}\left(\mathbb{N}, \mathbb{R}^{\text {sxs }}\right)$ transforms system (5) into system (27). Then (27) is completely controllable. Moreover, if

$$
\left\|D^{-1}(k)\right\| \leq C e^{\mu k}
$$

then

$$
\alpha_{\bar{A}, \bar{B}}\left(k_{0}, k_{0}+K\right) \geq C^{-2} e^{-2 \mu k_{0}} \alpha_{A, B}\left(k_{0}, k_{0}+K\right) .
$$

Proof Formula (28) implies that

$$
W_{\bar{A}, \bar{B}}\left(k_{0}, k_{0}+K\right)=D\left(k_{0}\right) W_{A, B}\left(k_{0}, k_{0}+K\right) D^{T}\left(k_{0}\right)
$$

and therefore, by Theorem 2, complete controllability (5) is equivalent to complete controllability of (27). Since for any symmetric matrix $V \in \mathbb{R}^{s \times s}$ and any matrix $D \in \mathbb{R}^{s \times s}$, we have

$$
\min _{x \in \mathbb{R}^{s}, x \neq 0} \frac{x^{T} V x}{x^{T} x}=\lambda_{\min }(V)
$$

and

$$
\lambda_{\min }(V)=\lambda_{\max }^{-1}\left(V^{-1}\right),\|D\|^{2}=\lambda_{\max }\left(D D^{T}\right)=\lambda_{\max }\left(D^{T} D\right)
$$

(see [49]), where $\lambda_{\min }(V)$ and $\lambda_{\max }(V)$ are the smallest and the greatest eigenvalue of $V$, respectively. Then using (32) with $V=W_{A, B}\left(k_{0}, k_{0}+K\right)$, we get

$$
\alpha_{A, B}\left(k_{0}, k_{0}+K\right) \leq \frac{x^{T} D^{-1}\left(k_{0}\right) W_{\bar{A}, \bar{B}}\left(k_{0}, k_{0}+K\right) D^{-T}\left(k_{0}\right) x}{x^{T} x} .
$$

Denoting $y=D^{-T}\left(k_{0}\right) x$ and having in mind that $D^{-T}\left(k_{0}\right)$ is invertible, we have

$$
\alpha_{A, B}\left(k_{0}, k_{0}+K\right) \leq \frac{y^{T} W_{\bar{A}, \bar{B}}\left(k_{0}, k_{0}+K\right) y}{y^{T} D\left(k_{0}\right) D^{T}\left(k_{0}\right) y}
$$

for any $y \in \mathbb{R}^{s}, y \neq 0$. Using (32) with $V=D\left(k_{0}\right) D^{T}\left(k_{0}\right)$ and (33) with $D=D\left(k_{0}\right)$, we get

$$
\begin{aligned}
y^{T} D\left(k_{0}\right) D^{T}\left(k_{0}\right) y & \geq \lambda_{\min }\left(D\left(k_{0}\right) D^{T}\left(k_{0}\right)\right) y^{T} y \\
& =\lambda_{\max }^{-1}\left(D^{-T}\left(k_{0}\right) D^{-1}\left(k_{0}\right)\right)=\left\|D^{-1}\left(k_{0}\right)\right\|^{-2} .
\end{aligned}
$$

Using the last inequality in (34), we obtain

$$
\alpha_{A, B}\left(k_{0}, k_{0}+K\right) \leq \frac{y^{T} W_{\bar{A}, \bar{B}}\left(k_{0}, k_{0}+K\right) y}{y^{T} y}\left\|D^{-1}\left(k_{0}\right)\right\|^{2}
$$

and taking into account (29), we have

$$
\alpha_{A, B}\left(k_{0}, k_{0}+K\right) \leq \frac{y^{T} W_{\bar{A}, \bar{B}}\left(k_{0}, k_{0}+K\right) y}{y^{T} y} C^{2} e^{2 \mu k_{0}} .
$$


Taking the infimum over $y \in \mathbb{R}^{s}, y \neq 0$ and using (32), we get

$$
\alpha_{A, B}\left(k_{0}, k_{0}+K\right) \leq \alpha_{\bar{A}, \bar{B}}\left(k_{0}, k_{0}+K\right) C^{2} e^{2 \mu k_{0}} .
$$

The last inequality implies (30).

Denote

$$
F(n, m)=\Phi_{A}(n, m+1) B(m) .
$$

Theorem 8 For any $K, k_{0} \in \mathbb{N}$ and $\xi \in \mathbb{R}^{s},\|\xi\|=1$, there exist $v \in \mathbb{R}^{t},\|v\|=1$ and $k_{v} \in \mathbb{N}$, $k_{0} \leq k_{v} \leq k_{0}+K-1$, such that

$$
\left|\xi^{T} F\left(k_{0}, k_{v}\right) v\right| \geq \sqrt{\frac{1}{t K} \alpha_{A, B}\left(k_{0}, k_{0}+K\right)}
$$

Proof First, we show that for any $k_{0}, K \in \mathbb{N}$ and $\xi \in \mathbb{R}^{s},\|\xi\|=1$, there exists $v \in \mathbb{R}^{t},\|v\|=1$ such that

$$
\sum_{j=k_{0}}^{k_{0}+K-1}\left(\xi^{T} F\left(k_{0}, j\right) \nu\right)^{2} \geq \frac{1}{t} \alpha_{A, B}\left(k_{0}, k_{0}+K\right)
$$

Since,

$$
W_{A, B}\left(k_{0}, k_{0}+K\right) \geq \alpha_{A, B}\left(k_{0}, k_{0}+K\right) I_{s}
$$

using the notation (35), we get

$$
\xi^{T} W_{A, B}\left(k_{0}, k_{0}+K\right) \xi=\sum_{j=k_{0}}^{k_{0}+K-1}\left\|\xi^{T} F\left(k_{0}, j\right)\right\|^{2} \geq \alpha_{A, B}\left(k_{0}, k_{0}+K\right)
$$

for any $k_{0}, K \in \mathbb{N}$ and any $\xi \in \mathbb{R}^{s},\|\xi\|=1$.

Let us fix $\xi \in \mathbb{R}^{s},\|\xi\|=1$, and define as $v$ as one of the vectors of the standard basis $e_{i}$, $i \in\{1, \ldots, t\}$, of $\mathbb{R}^{t}$ for which the expression

$$
\sum_{j=k_{0}}^{k_{0}+K-1}\left(\xi^{T} F\left(k_{0}, j\right) e_{i}\right)^{2}
$$

achieves its maximum. Then we have

$$
\begin{aligned}
t \sum_{j=k_{0}}^{k_{0}+K-1}\left(\xi^{T} F\left(k_{0}, j\right) \nu\right)^{2} & \geq \sum_{i=1}^{t} \sum_{j=k_{0}}^{k_{0}+K-1}\left(\xi^{T} F\left(k_{0}, j\right) e_{i}\right)^{2} \\
& =\sum_{j=k_{0}}^{k_{0}+K-1}\left\|\xi^{T} F\left(k_{0}, j\right)\right\|^{2} \geq \alpha_{A, B}\left(k_{0}, k_{0}+K\right) .
\end{aligned}
$$

The last inequality implies (37). From (37), the inequality (36) follows immediately. 
For an $s$ by $s$ matrix $H=\left[h_{i j}\right]_{i, j=1, \ldots, s}$, denote by $(H)_{k}$ the $k$ th leading minor, i.e.,

$$
(H)_{1}=h_{11}, \quad(H)_{2}=\left[\begin{array}{ll}
h_{11} & h_{12} \\
h_{21} & h_{22}
\end{array}\right], \quad \ldots, \quad(H)_{s}=H .
$$

For $0<\delta \leq 1$ and $r \geq 0$, denote

$$
\mathcal{H}(r, \delta)=\left\{H \in \mathbb{R}^{s \times s}:\left\|H-I_{s}\right\|<r, \operatorname{det}(H)_{j} \geq \delta, j=1, \ldots, s\right\},
$$

and by $\mathcal{H}$ the set of all $s$ by $s$ matrices with leading minors with positive determinants. The proof of the next lemma may be found in [29].

Lemma 5 A matrix $H$ belongs to $\mathcal{H}$ if and only if $H=L G$, where $L$ and $G$ are lower and upper triangular matrices with positive diagonal elements, respectively.

Let us introduce the following notation

$$
a\left(k_{0}, K\right)=\max \left\{\max \left\{\left\|A^{-1}(l)\right\|, 1\right\}: l=k_{0}, \ldots, k_{0}+K-1\right\}
$$

and

$$
b\left(k_{0}, K\right)=\max \left\{\|B(l)\|: l=k_{0}, \ldots, k_{0}+K-1\right\} .
$$

In our further considerations, we will use the following lemma.

Lemma 6 Suppose that system (5) is $K$-completely controllable. Then for any $k_{0} \in \mathbb{N}$, there exist $\eta_{i} \in \mathbb{R}^{t},\left\|\eta_{i}\right\|=1$, and $m_{i} \in \mathbb{N}, i=1, \ldots$, s, such that $k_{0} \leq m_{1} \leq \cdots \leq m_{s} \leq k_{0}+K-1$ and the matrix

$$
F\left(k_{0}\right)=\left[F\left(k_{0}, m_{1}\right) \eta_{1}, \ldots, F\left(k_{0}, m_{s}\right) \eta_{s}\right]
$$

is invertible, and

$$
\begin{aligned}
& \left\|F\left(k_{0}\right)\right\| \leq \sqrt{s} b\left(k_{0}, K\right)\left(a\left(k_{0}, K\right)\right)^{K}, \\
& \left\|F^{-1}\left(k_{0}\right)\right\| \leq \frac{\left(\sqrt{s} b\left(k_{0}, K\right)\left(a\left(k_{0}, K\right)\right)^{K}\right)^{s-1}}{\left(\sqrt{\frac{1}{t K} \alpha_{A, B}\left(k_{0}, k_{0}+K\right)}\right)^{s}} .
\end{aligned}
$$

Proof According to Theorem 8 , for any $k_{0} \in \mathbb{N}$ and $\xi \in \mathbb{R}^{s},\|\xi\|=1$, there exist $v \in \mathbb{R}^{t},\|v\|=$ 1 and $k_{v} \in \mathbb{N}, k_{0} \leq k_{v} \leq k_{0}+K-1$ such that (36) holds. Let us fix $k_{0} \in \mathbb{N}$. The construction will be done by induction. In the first step, consider any $\xi_{1} \in \mathbb{R}^{s}$, $\left\|\xi_{1}\right\|=1$. Then we find $v_{1} \in \mathbb{R}^{t},\left\|v_{1}\right\|=1$ and $k_{1} \in \mathbb{N}, k_{0} \leq k_{1} \leq k_{0}+K-1$, such that

$$
\left|\xi_{1}^{T} F\left(k_{0}, k_{1}\right) v_{1}\right| \geq \sqrt{\frac{1}{t K} \alpha_{A, B}\left(k_{0}, k_{0}+K\right)}
$$


Suppose that after the $d$ th step, we have $\xi_{i} \in \mathbb{R}^{s},\left\|\xi_{i}\right\|=1, v_{i} \in \mathbb{R}^{t},\left\|v_{i}\right\|=1$ and $k_{i} \in \mathbb{N}$, $k_{0} \leq k_{i} \leq k_{0}+K-1, i=1,2, \ldots, d$ such that

$$
\left|\xi_{i}^{T} F\left(k_{0}, k_{i}\right) v_{i}\right| \geq \sqrt{\frac{1}{t K} \alpha_{A, B}\left(k_{0}, k_{0}+K\right)}
$$

Denote by $M_{d}$ the orthogonal complement of

$$
\left\{\sum_{i=1}^{d} \alpha_{i} F\left(k_{0}, k_{i}\right) v_{i}: \alpha_{i} \in \mathbb{R}, i=1,2, \ldots, d\right\}
$$

in $\mathbb{R}^{s}$. Since $\operatorname{dim} M_{d} \geq s-d$, it follows that the set $M_{d}$ is not empty for $d \leq s-1$.

In the $(d+1)$-st step, we take any $\xi_{d+1} \in M_{d},\left\|\xi_{d+1}\right\|=1$, and for it, we define $v_{d+1} \in \mathbb{R}^{t}$, $\left\|v_{d+1}\right\|=1$ and $k_{d+1} \in \mathbb{N}, k_{0} \leq k_{d+1} \leq k_{0}+K-1$ such that

$$
\left|\xi_{d+1}^{T} F\left(k_{0}, k_{d+1}\right) v_{d+1}\right| \geq \sqrt{\frac{1}{t K} \alpha_{A, B}\left(k_{0}, k_{0}+K\right)} .
$$

After $s$ steps, we will have $s$ vectors $\xi_{i}, i=1, \ldots, s$, such that the inequality (41) holds for all $i=1, \ldots, s$ and

$$
\xi_{i}^{T} F\left(k_{0}, k_{j}\right) v_{j}=0
$$

for all $i>j$. Denote

$$
S=\left[\xi_{1}, \ldots, \xi_{s}\right], \quad \widehat{F}\left(k_{0}\right)=\left[F\left(k_{0}, k_{1}\right) v_{1}, \ldots, F\left(k_{0}, k_{s}\right) v_{s}\right] .
$$

From the construction, it follows that the matrix $P=S^{T} \widehat{F}\left(k_{0}\right)$ is upper triangular and the diagonal elements $p_{i i}$ satisfy

$$
\left|p_{i i}\right| \geq \sqrt{\frac{1}{t K} \alpha_{A, B}\left(k_{0}, k_{0}+K\right)} ;
$$

therefore,

$$
|\operatorname{det} P| \geq\left(\sqrt{\frac{1}{t K} \alpha_{A, B}\left(k_{0}, k_{0}+K\right)}\right)^{s}>0
$$

and in particular $\operatorname{det} S \neq 0$. Moreover, by Hadamard's inequality (see [49, p. 477]), we have

$$
|\operatorname{det} S| \leq\left\|\xi_{1}\right\| \cdots\left\|\xi_{s}\right\|=1
$$

and consequently

$$
\left|\operatorname{det} \widehat{F}\left(k_{0}\right)\right|=\frac{|\operatorname{det} P|}{|\operatorname{det} S|} \geq\left(\sqrt{\frac{1}{t K} \alpha_{A, B}\left(k_{0}, k_{0}+K\right)}\right)^{s} .
$$

By [49, p. 313]

$$
\|\Gamma\| \leq\left(\sum_{i=1}^{s}\left\|\Gamma e_{i}\right\|^{2}\right)^{1 / 2}
$$


for any matrix $\Gamma \in \mathbb{R}^{s \times s}$, and we have

$$
\begin{aligned}
\left\|\widehat{F}\left(k_{0}\right)\right\| & \leq\left(\sum_{i=1}^{s}\left\|\widehat{F}\left(k_{0}\right) e_{i}\right\|^{2}\right)^{1 / 2} \\
& \leq \sqrt{s} \max _{i=1, \ldots, s}\left\|\widehat{F}\left(k_{0}\right) e_{i}\right\| \leq \sqrt{s} b\left(k_{0}, K\right)\left(a\left(k_{0}, K\right)\right)^{K} \\
\left\|\widehat{F}^{-1}\left(k_{0}\right)\right\| & \leq \frac{\left\|\widehat{F}\left(k_{0}\right)\right\|^{s-1}}{\left|\operatorname{det} \widehat{F}\left(k_{0}\right)\right|} \leq \frac{\left(\sqrt{s} b\left(k_{0}, K\right)\left(a\left(k_{0}, K\right)\right)^{K}\right)^{s-1}}{\left(\sqrt{\frac{1}{t K} \alpha_{A, B}\left(k_{0}, k_{0}+K\right)}\right)^{s}} .
\end{aligned}
$$

Let us rearrange the sequence $k_{1}, k_{2}, \ldots, k_{s}$ such that it forms a nondecreasing sequence. The elements of the new sequence will be denoted by $m_{i}$. So, we have

$$
k_{0} \leq m_{1} \leq \cdots \leq m_{s} \leq k_{0}+K-1, \quad i=1,2, \ldots, s
$$

In the same way, we rearrange the vectors $v_{1}, \ldots, v_{s}$ to obtain vectors $\eta_{1}, \ldots, \eta_{s}$. Then the matrix

$$
F\left(k_{0}\right)=\left[F\left(k_{0}, m_{1}\right) \eta_{1}, \ldots, F\left(k_{0}, m_{s}\right) \eta_{s}\right]
$$

which is obtained from $\widehat{F}\left(k_{0}\right)$ by swapping columns accordingly, satisfies (39) and (40).

The next theorem shows that by a special choice of control, we may connect the transition matrices of (1) and (7) by a particular relation.

Theorem 9 Suppose that system (5) is $K$-completely controllable. Then for any $k_{0} \in \mathbb{N}$, there exists an invertible s by s matrix $\Psi\left(k_{0}\right)$ with the following property: for any $0<\delta \leq 1$ and $r \geq 0$, there exist $\beta_{1}\left(r, \delta, k_{0}\right)>0$ and $\beta_{2}\left(r, \delta, k_{0}\right)>0$ such that for any $H \in \mathcal{H}(r, \delta)$, there exist a feedback control

$$
U=(U(i))_{i=k_{0}, \ldots, k_{0}+K-1}
$$

such that

$$
\Phi_{A+B U}\left(k_{0}+K, k_{0}\right)=\Phi_{A}\left(k_{0}+K, k_{0}\right) \Psi\left(k_{0}\right) H \Psi^{-1}\left(k_{0}\right)
$$

where $\Phi_{A+B U}$ is the transition matrix of the closed loop system (7) and

$$
\begin{gathered}
\max _{i=k_{0}, \ldots, k_{0}+K-1}\|U(i)\| \leq \beta_{1}\left(r, \delta, k_{0}\right)\left\|H-I_{s}\right\|, \\
\max _{i=k_{0}, \ldots, k_{0}+K-1}\left\|(A(i)+B(i) U(i))^{-1}\right\| \leq \beta_{2}\left(r, \delta, k_{0}\right),
\end{gathered}
$$

where

$$
\beta_{1}\left(r, \delta, k_{0}\right)=\frac{\sqrt{s}(1+r s)^{s-1}}{b\left(k_{0}, K\right) \delta}\left(\frac{\sqrt{s} b\left(k_{0}, K\right)\left(a\left(k_{0}, K\right)\right)^{K}}{\sqrt{\frac{1}{t K} \alpha_{A, B}\left(k_{0}, k_{0}+K\right)}}\right)^{2 s},
$$




$$
\begin{aligned}
& \beta_{2}\left(r, \delta, k_{0}\right)=\rho\left(a\left(k_{0}, K\right)\right)^{2 K}(1+r s)\left(\frac{\sqrt{s} b\left(k_{0}, K\right)\left(a\left(k_{0}, K\right)\right)^{K}}{\sqrt{\frac{1}{t K} \alpha_{A, B}\left(k_{0}, k_{0}+K\right)}}\right)^{s}, \\
& \rho=\left(\frac{\sqrt{s} b\left(k_{0}, K\right)\left(a\left(k_{0}, K\right)\right)^{K}}{\sqrt{\frac{1}{t K} \alpha_{A, B}\left(k_{0}, k_{0}+K\right)}}\right)^{s} \frac{(1+r s)^{s-1}}{\delta} \\
& \left\|\Psi\left(k_{0}\right)\right\| \leq \sqrt{s} b\left(k_{0}, K\right)\left(a\left(k_{0}, K\right)\right)^{K}
\end{aligned}
$$

and

$$
\left\|\Psi^{-1}\left(k_{0}\right)\right\| \leq \frac{\left(\sqrt{s} b\left(k_{0}, K\right)\left(a\left(k_{0}, K\right)\right)^{K}\right)^{s-1}}{\left(\sqrt{\frac{1}{t K} \alpha_{A, B}\left(k_{0}, k_{0}+K\right)}\right)^{s}} .
$$

Proof Let us fix $k_{0} \in \mathbb{N}$, and let $\eta_{i} \in \mathbb{R}^{t}, m_{i} \in \mathbb{N}, i=1, \ldots, s$, and $\Psi\left(k_{0}\right)=F\left(k_{0}\right)$ be as in Lemma 6. Consider any $0<\delta \leq 1, r \geq 0, H \in \mathcal{H}(r, \delta)$ and the matrix equations

$$
Y(k+1)=A(k) Y(k)+B(k) V(k), \quad k=k_{0}, k_{0}+1, \ldots, k_{0}+K-1,
$$

where $Y(k)$ is a $s$ by $s$, and $V(k)$ is a $t$ by $s$ matrix. We will consider this equation with initial condition

$$
Y\left(k_{0}\right)=I_{s},
$$

and we construct a sequence $V(k), k=k_{0}, k_{0}+1, \ldots, k_{0}+K-1$, such that

$$
Y\left(k_{0}+K\right)=\Phi_{A}\left(k_{0}+K, k_{0}\right) \Psi\left(k_{0}\right) H \Psi^{-1}\left(k_{0}\right)
$$

The solution of equation (44), with the considered initial condition, is given by

$$
Y(k)=\Phi_{A}\left(k, k_{0}\right)\left[I_{s}+\sum_{j=k_{0}}^{k-1} \Phi_{A}\left(k_{0}, j+1\right) B(j) V(j)\right]
$$

and the condition (45) will be satisfied if and only if

$$
I_{s}+\sum_{j=k_{0}}^{k_{0}+K-1} \Phi_{A}\left(k_{0}, j+1\right) B(j) V(j)=\Psi\left(k_{0}\right) H \Psi^{-1}\left(k_{0}\right) .
$$

Denote

$$
\begin{aligned}
& G(k)=I_{s}+\sum_{j=k_{0}}^{k-1} \Phi_{A}\left(k_{0}, j+1\right) B(j) V(j)=I_{s}+\sum_{j=k_{0}}^{k-1} F\left(k_{0}, j\right) V(j), \\
& I_{j}=\left\{i \in \mathbb{N}: m_{i}=j\right\}, \text { and } \bar{I}_{j}=\left\{i \in \mathbb{N}: m_{i} \leq j-1\right\} .
\end{aligned}
$$

In the further calculation, we will consider sums of the form $\sum_{i \in I_{j}}$ and $\sum_{i \in \bar{I}_{j}}$. In the case when $I_{j}$ or $\bar{I}_{j}$ is empty, we define the sum as equal to zero. 
Set

$$
u_{i}=\Psi^{-T}\left(k_{0}\right)\left(H^{T}-I_{s}\right) e_{i}, \quad V(j)=\sum_{i \in I_{j}} \eta_{i} u_{i}^{T}
$$

where $e_{i}, i=1, \ldots, s$, is the standard basis of $\mathbb{R}^{s}$, and $\eta_{i}$ are defined in Lemma 6 , equation (38).

We have

$$
\begin{aligned}
\sup _{k_{0} \leq k \leq k_{0}+K-1}\|V(k)\| & \leq \sup _{k_{0} \leq k \leq k_{0}+K-1} \sum_{i \in I_{k}}\left\|\eta_{i}\right\|\left\|u_{i}^{T}\right\| \leq s\left\|\Psi\left(k_{0}\right)^{-1}\right\|\left\|H-I_{s}\right\| \\
& \leq s \frac{\left(\sqrt{s} b\left(k_{0}, K\right)\left(a\left(k_{0}, K\right)\right)^{K}\right)^{s-1}}{\left(\sqrt{\frac{1}{t K} \alpha_{A, B}\left(k_{0}, k_{0}+K\right)}\right)^{s}}\left\|H-I_{s}\right\|,
\end{aligned}
$$

where

$$
\gamma=s \frac{\left(\sqrt{s} b\left(k_{0}, K\right)\left(a\left(k_{0}, K\right)\right)^{K}\right)^{s-1}}{\left(\sqrt{\frac{1}{t K} \alpha_{A, B}\left(k_{0}, k_{0}+K\right)}\right)^{s}}
$$

and

$$
\begin{aligned}
G(k) & =I_{s}+\sum_{j=k_{0}}^{k-1} F\left(k_{0}, j\right) \sum_{i \in I_{j}} \eta_{i} u_{i}^{T}=I_{s}+\sum_{i \in \overline{I_{k}}} F\left(k_{0}\right) e_{i} u_{i}^{T} \\
& =I_{s}+\sum_{i \in \overline{I_{k}}} F\left(k_{0}\right) e_{i} e_{i}^{T}\left(H-I_{s}\right) F^{-1}\left(k_{0}\right) \\
& =F\left(k_{0}\right)\left(I_{s}+\sum_{i \in \overline{I_{k}}} e_{i} e_{i}^{T}\left(H-I_{s}\right)\right) F^{-1}\left(k_{0}\right)=\Psi\left(k_{0}\right) S_{k} \Psi^{-1}\left(k_{0}\right),
\end{aligned}
$$

where the step matrices $S_{k}$ are defined as follows

$$
S_{k}=I_{s}+\sum_{i \in \overline{I_{k}}} e_{i} e_{i}^{T}\left(H-I_{s}\right)
$$

and have the form

$$
\left.\left[\begin{array}{c}
H \\
I_{s-q}
\end{array}\right]\right\} \begin{aligned}
& q \text { rows } \\
& (s-q) \text { rows }
\end{aligned}
$$

with $q=\max \left\{i: m_{i} \leq k-1\right\}$. Then

$$
G^{-1}(k)=\Psi\left(k_{0}\right) S_{k}^{-1} \Psi^{-1}\left(k_{0}\right)
$$

and

$$
\left\|G^{-1}(k)\right\| \leq\left\|\Psi\left(k_{0}\right)\right\|\left\|\Psi^{-1}\left(k_{0}\right)\right\|\left\|S_{k}^{-1}\right\|
$$




$$
\begin{aligned}
& \leq \sqrt{s} b\left(k_{0}, K\right)\left(a\left(k_{0}, K\right)\right)^{K} \frac{\left(\sqrt{s} b\left(k_{0}, K\right)\left(a\left(k_{0}, K\right)\right)^{K}\right)^{s-1}}{\left(\sqrt{\frac{1}{t K} \alpha_{A, B}\left(k_{0}, k_{0}+K\right)}\right)^{s}}\left\|S_{k}^{-1}\right\| \\
& \leq \frac{\left(\sqrt{s} b\left(k_{0}, K\right)\left(a\left(k_{0}, K\right)\right)^{K}\right)^{s}}{\left(\sqrt{\frac{1}{t K} \alpha_{A, B}\left(k_{0}, k_{0}+K\right)}\right)^{s}} \frac{\left\|S_{k}\right\|^{s-1}}{\operatorname{det} S_{k}} \\
& \leq\left(\frac{\sqrt{s} b\left(k_{0}, K\right)\left(a\left(k_{0}, K\right)\right)^{K}}{\sqrt{\frac{1}{t K} \alpha_{A, B}\left(k_{0}, k_{0}+K\right)}}\right)^{s} \frac{(1+r s)^{s-1}}{\delta}=: \rho .
\end{aligned}
$$

Because of Lemma 6, we know that

$$
\operatorname{det} S_{k}=\operatorname{det} H_{q} \geq \delta,
$$

and therefore,

$$
\left\|S_{k}\right\|=\left\|I_{s}+\sum_{i \in \overline{I_{k}}} e_{j} e_{j}^{T}\left(H-I_{s}\right)\right\| \leq 1+\left\|H-I_{s}\right\| \sum_{i \in \overline{I_{k}}}\left\|e_{j} e_{j}^{T}\right\| \leq 1+r s,
$$

since $H \in \mathcal{H}(r, \delta)$.

We have constructed matrices $V(k), k=k_{0}, \ldots, k_{0}+K-1$, such that the matrix

$$
G(k)=I_{s}+\sum_{j=k_{0}}^{k-1} \Phi_{A}\left(k_{0}, j+1\right) B(j) V(j)
$$

is invertible for any $k=k_{0}, \ldots, k_{0}+K-1$, and such that

$$
\|V(k)\| \leq \gamma\left\|H-I_{s}\right\|, \quad\left\|G^{-1}(k)\right\| \leq \rho,
$$

where

$$
\gamma=s \frac{\left(\sqrt{s} b\left(k_{0}, K\right)\left(a\left(k_{0}, K\right)\right)^{K}\right)^{s-1}}{\left(\sqrt{\frac{1}{t K} \alpha_{A, B}\left(k_{0}, k_{0}+K\right)}\right)^{s}},
$$

and

$$
\rho=\left(\frac{\sqrt{s} b\left(k_{0}, K\right)\left(a\left(k_{0}, K\right)\right)^{K}}{\sqrt{\frac{1}{t K} \alpha_{A, B}\left(k_{0}, k_{0}+K\right)}}\right)^{s} \frac{(1+r s)^{s-1}}{\delta} .
$$

From the construction of the control sequence, it follows that the matrix

$$
Y(k)=\Phi_{A}\left(k_{0}, k\right) G(k)
$$

is invertible for any $k=k_{0}, \ldots, k_{0}+K-1$, and

$$
\left\|Y^{-1}(k)\right\| \leq \rho\left(a\left(k_{0}, K\right)\right)^{K} .
$$

Taking

$$
U(k)=V(k) Y^{-1}(k),
$$


we get

$$
Y(k+1)=A(k) Y(k)+B(k) V(k)=(A(k)+B(k) U(k)) Y(k)
$$

and because $Y\left(k_{0}\right)=I_{s}$, the equality (42) holds. Moreover,

$$
\|U(k)\| \leq\|V(k)\|\left\|Y^{-1}(k)\right\| \leq \beta_{1}\left(r, \delta, k_{0}\right)\left\|H-I_{s}\right\|,
$$

where

$$
\begin{aligned}
\beta_{1}\left(r, \delta, k_{0}\right)= & \gamma \rho a\left(k_{0}, K\right)^{K} \\
= & s \frac{\left(\sqrt{s} b\left(k_{0}, K\right)\left(a\left(k_{0}, K\right)\right)^{K}\right)^{s-1}}{\left(\frac{1}{t K} \alpha_{A, B}\left(k_{0}, k_{0}+K\right)\right)^{s}} \\
& \times\left(\frac{\sqrt{s} b\left(k_{0}, K\right)\left(a\left(k_{0}, K\right)\right)^{K}}{\sqrt{\frac{1}{t K} \alpha_{A, B}\left(k_{0}, k_{0}+K\right)}}\right)^{s} \frac{(1+r s)^{s-1}}{\delta} a\left(k_{0}, K\right)^{K} \\
= & \frac{s}{\sqrt{s} b\left(k_{0}, K\right)}\left(\frac{\sqrt{t K s} b\left(k_{0}, K\right)\left(a\left(k_{0}, K\right)\right)^{K}}{\sqrt{\frac{1}{t K} \alpha_{A, B}\left(k_{0}, k_{0}+K\right)}}\right)^{2 s} \frac{(1+r s)^{s-1}}{\delta} .
\end{aligned}
$$

Observe that for any $k \in\left\{k_{0}, k_{0}+1, \ldots, k_{0}+K-1\right\}$, we have

$$
(A(k)+B(k) U(k))^{-1}=Y(k) Y^{-1}(k+1) .
$$

This implies

$$
\begin{aligned}
\left\|(A(k)+B(k) U(k))^{-1}\right\| \leq & \|Y(k)\|\left\|Y^{-1}(k+1)\right\| \\
\leq & \left\|\Phi_{A}\left(k_{0}, k\right)\right\|\|G(k)\|\left\|Y^{-1}(k+1)\right\| \\
\leq & \left(a\left(k_{0}, K\right)\right)^{K}\left\|\Psi\left(k_{0}\right)\right\|\left\|S_{k}\right\|\left\|\Psi^{-1}\left(k_{0}\right)\right\| \rho\left(a\left(k_{0}, K\right)\right)^{K} \\
\leq & \left(a\left(k_{0}, K\right)\right)^{K} \sqrt{s} b\left(k_{0}, K\right)\left(a\left(k_{0}, K\right)\right)^{K} \\
& \times(1+r s) \frac{\left(\sqrt{s} b\left(k_{0}, K\right)\left(a\left(k_{0}, K\right)\right)^{K}\right)^{s-1}}{\left(\sqrt{\frac{1}{t K} \alpha_{A, B}\left(k_{0}, k_{0}+K\right)}\right)^{s}} \rho\left(a\left(k_{0}, K\right)\right)^{K} \\
= & \rho\left(a\left(k_{0}, K\right)\right)^{2 K}(1+r s)\left(\frac{\sqrt{t K s} b\left(k_{0}, K\right)\left(a\left(k_{0}, K\right)\right)^{K}}{\sqrt{\frac{1}{t K} \alpha_{A, B}\left(k_{0}, k_{0}+K\right)}}\right)^{s} \\
= & \beta_{2}(r, \delta) .
\end{aligned}
$$

Theorem 10 Suppose that system (5) is K-completely controllable, then there exists a sequence $(T(k))_{k \in \mathbb{N}}, T(k) \in \mathrm{GL}_{s}(\mathbb{R})$, such that

$$
\begin{aligned}
& \|T(k)\| \leq \sqrt{s} b((k-1) K, K)(a((k-1) K, K))^{K}, \\
& \left\|T^{-1}(k)\right\| \leq \frac{\left(\sqrt{s} b((k-1) K, K)(a((k-1) K, K))^{K}\right)^{s-1}}{\left(\sqrt{\frac{1}{t K} \alpha_{A, B}((k-1) K, k K)}\right)^{s}},
\end{aligned}
$$


and for each upper triangular sequence $(L(k))_{k \in \mathbb{N}}, L(k) \in \mathrm{GL}_{s}(\mathbb{R})$ and each lower triangular sequence $(G(k))_{k \in \mathbb{N}}, G(k) \in \mathrm{GL}_{s}(\mathbb{R})$, of matrices with positive diagonal elements (both $L$ and $G)$, there exists a feedback control $U=(U(k))_{k \in \mathbb{N}}$ such that

$$
\begin{aligned}
& \Phi_{A+B U}(k K,(k-1) K)=\Phi_{A}(k K,(k-1) K) T(k) L(k) G(k) T^{-1}(k), \\
& \|U(k)\| \leq\left(\gamma^{2}(k)+1\right) \beta_{1}\left(\gamma^{2}(k)+1, \gamma^{-2 s}(k),(k-1) K\right)
\end{aligned}
$$

and

$$
\left\|(A(k)+B(k) U(k))^{-1}\right\| \leq \beta_{2}\left(\gamma^{2}(k)+1, \gamma^{-2 s}(k),(k-1) K\right),
$$

where

$$
\gamma(k)=\max \left\{\|L(k)\|,\left\|L^{-1}(k)\right\|,\|G(k)\|,\left\|G^{-1}(k)\right\|\right\} .
$$

Proof Consider sequences $(L(k))_{k \in \mathbb{N}}$ and $(G(k))_{k \in \mathbb{N}}$ as in the theorem. Then for the diagonal elements $l_{i i}(k), i=1, \ldots, s$, of the matrix $L(k)$, we have

$$
\begin{aligned}
& l_{i i}(k)=\left|l_{i i}(k)\right|=\left|e_{i}^{T} L(k) e_{i}\right| \leq\|L(k)\| \leq \gamma(k), \\
& 1 / l_{i i}(k)=\left|1 / l_{i i}(k)\right|=\left|e_{i}^{T} L^{-1}(k) e_{i}\right| \leq\left\|L^{-1}(k)\right\| \leq \gamma(k)
\end{aligned}
$$

for all $k \in \mathbb{N}$. From the last two inequalities, we obtain

$$
\frac{1}{\gamma(k)} \leq l_{i i}(k) \leq \gamma(k), \quad i=1, \ldots, s, k \in \mathbb{N} .
$$

The same estimates can be obtained for the diagonal elements $g_{i i}(k), i=1, \ldots, s$, of the matrix $G(k)$. Therefore,

$$
\operatorname{det}(L(k))_{j}=\prod_{i=1}^{j} l_{i i}(k) \geq \gamma^{-j}(k), \quad \operatorname{det}(G(k))_{j}=\prod_{i=1}^{j} g_{i i}(k) \geq \gamma^{-j}(k)
$$

for all $k \in \mathbb{N}$ and $j=1, \ldots, s$, where $(H)_{j}$ is the $j$ th leading minor of the matrix $H$.

Let $H(k)=L(k) G(k), k \in \mathbb{N}$. From Lemma 5, we know that $H(k) \in \mathcal{H}$; moreover,

$$
\begin{aligned}
& \left\|H(k)-I_{s}\right\| \leq\|H(k)\|+1 \leq\|L(k)\|\|G(k)\|+1 \leq \gamma^{2}(k)+1, \\
& \operatorname{det}(H(k))_{j}=\operatorname{det}(L(k))_{j} \operatorname{det}(G(k))_{j} \geq \gamma^{-2 j}(k) \geq \gamma^{-2 s}(k), \quad j=1, \ldots, s .
\end{aligned}
$$

Therefore,

$$
H(k) \in \mathcal{H}\left(\gamma^{2}(k)+1, \gamma^{-2 s}(k)\right) .
$$

From Theorem 9, we conclude that for each $k \in \mathbb{N}$, there exist control sequences $U_{k}(l)$, $l=(k-1) K, \ldots, k K-1$, and an invertible $s$ by $s$ matrix $\Psi((k-1) K)$ such that

$$
\max _{l=(k-1) K, \ldots, k K-1}\left\|U_{k}(l)\right\| \leq \beta\left(\gamma^{2}(k)+1, \gamma^{-2 s}(k)\right)\left\|H(k)-I_{s}\right\|
$$


and

$$
\Phi_{A+B U_{k}}(k K,(k-1) K)=\Phi_{A}(k K,(k-1) K) \Psi((k-1) K) H(k) \Psi^{-1}((k-1) K) .
$$

It follows from the proof of Theorem 9 that $\Psi((k-1) K)=F((k-1) K)$, where $F$ is defined by (38). Set

$$
T(k)=F((k-1) K), \quad k \in \mathbb{N} .
$$

From (39) and (40), it is clear that $(T(k))_{k \in \mathbb{N}}$ satisfies

$$
\begin{aligned}
& \|T(k)\| \leq \sqrt{s} b((k-1) K, K)(a((k-1) K, K))^{K}, \\
& \left\|T^{-1}(k)\right\| \leq \frac{\left(\sqrt{s} b((k-1) K, K)(a((k-1) K, K))^{K}\right)^{s-1}}{\left(\sqrt{\frac{1}{t K} \alpha_{A, B}((k-1) K, k K)}\right)^{s}} .
\end{aligned}
$$

Moreover,

$$
\Phi_{A+B U_{k}}(k K,(k-1) K)=\Phi_{A}(k K,(k-1) K) T(k) H(k) T^{-1}(k) .
$$

Let us define

$$
U(k)=U_{k}(k) \text { for } k=(k-1) K, \ldots, k K-1,
$$

then it is clear that

$$
\|U(k)\| \leq\left(\gamma^{2}(k)+1\right) \beta_{1}\left(\gamma^{2}(k)+1, \gamma^{-2 s}(k),(k-1) K\right)
$$

and (49) holds. Moreover, by (43), it follows that

$$
\left\|(A(k)+B(k) U(k))^{-1}\right\| \leq \beta_{2}\left(\gamma^{2}(k)+1, \gamma^{-2 s}(k),(k-1) K\right) .
$$

Condition (51) in the following corollary to Theorem 10 ensures the existence of a tempered transformation and a control in a specific situation. It will be useful to prove our main result.

Corollary 1 Suppose that $A, A^{-1} \in \mathcal{L}^{\mathrm{tem}}\left(\mathbb{N}, \mathbb{R}^{s \times s}\right), B \in \mathcal{L}^{\mathrm{tem}}\left(\mathbb{N}, \mathbb{R}^{s \times t}\right)$, system (5) is $K$ completely controllable and

$$
\liminf _{k \rightarrow \infty} \frac{1}{k} \ln \alpha_{A, B}((k-1) K, k K) \geq 0 .
$$

Then there exists a sequence $T=(T(k))_{k \in \mathbb{N}}, T, T^{-1} \in \mathcal{L}^{\operatorname{tem}}\left(\mathbb{N}, \mathbb{R}^{s \times s}\right), T(k) \in \mathrm{GL}_{s}(\mathbb{R}), k \in \mathbb{N}$ such that for each upper triangular sequence $(L(k))_{k \in \mathbb{N}},\left(L^{-1}(k)\right)_{k \in \mathbb{N}} \in \mathcal{L}^{\text {tem }}\left(\mathbb{N}, \mathbb{R}^{s \times s}\right), L(k) \in$ $\mathrm{GL}_{s}(\mathbb{R}), k \in \mathbb{N}$ and each lower triangular sequence $(G(k))_{k \in \mathbb{N}},\left(G^{-1}(k)\right)_{k \in \mathbb{N}} \in \mathcal{L}^{\mathrm{tem}}\left(\mathbb{N}, \mathbb{R}^{s \times s}\right)$, $G(k) \in \mathrm{GL}_{s}(\mathbb{R}), k \in \mathbb{N}$ of matrices with positive diagonal elements (both $L$ and $G$ ), there exists an admissible control $U=(U(k))_{k \in \mathbb{N}}$ such that

$$
\Phi_{A+B U}(k K,(k-1) K)=\Phi_{A}(k K,(k-1) K) T(k) L(k) G(k) T^{-1}(k) .
$$


Proof Let $T=(T(k))_{k \in \mathbb{N}}$ be the sequence from Theorem 10. First, we will show that $T$ and $T^{-1}$ are tempered. Since $A, A^{-1}$ and $B$ are tempered for each $\varepsilon>0$, there exists $C(\varepsilon)>0$ such that

$$
\left\|A^{-1}(k)\right\| \leq C(\varepsilon) e^{\varepsilon k}, \quad\|B(k)\| \leq C(\varepsilon) e^{\varepsilon k}
$$

and by (51)

$$
\alpha_{A, B}((k-1) K, k K) \geq \frac{1}{C(\varepsilon)} e^{-\varepsilon k}
$$

for all $k \in \mathbb{N}$. This implies that

$$
a((k-1) K, K) \leq C(\varepsilon) e^{\varepsilon k}, \quad b((k-1) K, K) \leq C(\varepsilon) e^{\varepsilon k}
$$

and

$$
\frac{1}{\alpha_{A, B}((k-1) K, k K)} \leq C(\varepsilon) e^{\varepsilon k} .
$$

From the last three inequalities, (46) and (47), we get

$$
\begin{aligned}
\|T(k)\| & \leq \sqrt{s} b((k-1) K, K)(a((k-1) K, K))^{K} \\
& \leq \sqrt{s} C(\varepsilon) e^{\varepsilon k} C^{K}(\varepsilon) e^{\varepsilon k K}=C_{1}\left(\varepsilon_{1}\right) e^{\varepsilon_{1} k}
\end{aligned}
$$

and

$$
\begin{aligned}
\left\|T^{-1}(k)\right\| & \leq \frac{\left(\sqrt{s} b((k-1) K, K)(a((k-1) K, K))^{K}\right)^{s-1}}{\left(\sqrt{\frac{1}{t K} \alpha_{A, B}((k-1) K, k K)}\right)^{s}} \\
& \leq \frac{\sqrt{s}}{\left(\sqrt{\frac{1}{t K}}\right)^{s}} C(\varepsilon) e^{\varepsilon k}\left(C(\varepsilon) e^{\varepsilon k}\right)^{K(s-1)} \sqrt{C(\varepsilon)} e^{\frac{1}{2} \varepsilon k} \\
& \leq \frac{\sqrt{s}}{\left(\sqrt{\frac{1}{t K}}\right)^{s}} C(\varepsilon)^{K(s-1)+1} e^{\left(K(s-1)+\frac{3}{2}\right) \varepsilon k}=C_{2}\left(\varepsilon_{2}\right) e^{\varepsilon_{2} k},
\end{aligned}
$$

where

$$
\begin{aligned}
& \varepsilon_{1}=(K+1) \varepsilon, \quad C_{1}\left(\varepsilon_{1}\right)=\sqrt{s} C(\varepsilon) C^{K}(\varepsilon) \\
& \varepsilon_{2}=\left(K(s-1)+\frac{3}{2}\right) \varepsilon \quad \text { and } \quad C_{2}\left(\varepsilon_{2}\right)=\frac{\sqrt{s}}{\left(\sqrt{\frac{1}{t K}}\right)^{s}} C^{K(s-1)+1}(\varepsilon) .
\end{aligned}
$$

From (55) and (56), it follows that $T$ and $T^{-1}$ are tempered since $\varepsilon_{1}$ and $\varepsilon_{2}$ tend to zero when $\varepsilon$ tends to zero. Let us fix $L$ and $G$ as in the assumptions of the theorem, and let $U=(U(k))_{k \in \mathbb{N}}$ be from Theorem 10. Then from Theorem 10, it follows that (52) holds, and therefore, $A(k)+B(k) U(k) \in \mathrm{GL}_{s}(\mathbb{R})$. Let us estimate $(\|U(k)\|)_{k \in \mathbb{N}}$. According to (49), we have

$$
\|U(k)\| \leq\left(\gamma^{2}(k)+1\right) \beta_{1}\left(\gamma^{2}(k)+1, \gamma^{-2 s}(k),(k-1) K\right) .
$$


Since $L, L^{-1}, G$ and $G^{-1}$ are tempered for each $\varepsilon>0$, there exists $C(\varepsilon)>0$ such that

$$
\gamma(k) \leq C_{3}(\varepsilon) e^{\varepsilon k}
$$

for all $k \in \mathbb{N}$, and therefore, by (53) and (54), we get

$$
\begin{aligned}
& \beta_{1}\left(\gamma^{2}(k)+1, \gamma^{-2 s}(k),(k-1) K\right) \\
& =\frac{\sqrt{s}\left(1+\left(\gamma^{2}(k)+1\right) s\right)^{s-1}}{b((k-1) K, K) \gamma^{-2 s}(k)}\left(\frac{\sqrt{s} b((k-1) K, K)(a((k-1) K, K))^{K}}{\sqrt{\frac{1}{t K} \alpha_{A, B}((k-1) K, k K)}}\right)^{2 s} \\
& =\frac{\sqrt{s}\left(1+\left(\gamma^{2}(k)+1\right) s\right)^{s-1}}{b((k-1) K, K) \gamma^{-2 s}(k)}\left(\frac{\sqrt{s} b((k-1) K, K)(a((k-1) K, K))^{K}}{\sqrt{\frac{1}{t K} \alpha_{A, B}((k-1) K, k K)}}\right)^{2 s} \\
& =\frac{(\sqrt{s})^{2 s+1}\left(1+\left(\gamma^{2}(k)+1\right) s\right)^{s-1} \gamma^{2 s}(k)}{\left(\sqrt{\frac{1}{t K}}\right)^{2 s}} \frac{(b((k-1) K, K))^{2 s-1}(a((k-1) K, K))^{2 s K}}{\alpha_{A, B}^{s}((k-1) K, k K)} \\
& \leq \frac{(\sqrt{s})^{2 s+1}\left(C_{3}^{2}(\varepsilon) s e^{2 \varepsilon k}+s+1\right)^{2 s-1} C_{3}^{2 s}(\varepsilon) e^{2 s \varepsilon k}}{\left(\sqrt{\frac{1}{t K}}\right)^{2 s}}\left(C(\varepsilon) e^{\varepsilon k}\right)^{2 s-1}\left(C(\varepsilon) e^{\varepsilon k}\right)^{2 s K}\left(C(\varepsilon) e^{\varepsilon k}\right)^{s} \\
& =\frac{(\sqrt{s})^{2 s+1}\left(C_{3}^{2}(\varepsilon) s e^{2 \varepsilon k}+s+1\right)^{2 s-1} C_{3}^{2 s}(\varepsilon)}{\left(\sqrt{\frac{1}{t K}}\right)^{2 s}} C^{3 s+2 s K-1}(\varepsilon) e^{\varepsilon k(5 s+2 s K-1)} .
\end{aligned}
$$

It is clear that there exists a constant $C_{4}(\varepsilon)>0$ such that

$$
C_{3}^{2}(\varepsilon) s e^{2 \varepsilon k}+s+1 \leq C_{4}(\varepsilon) e^{2 \varepsilon k}
$$

for all $k \in \mathbb{N}$. Using the last inequality in (59), we get

$$
\beta_{1}\left(\gamma^{2}(k)+1, \gamma^{-2 s}(k),(k-1) K\right) \leq C_{5}\left(\varepsilon_{5}\right) e^{\varepsilon_{5} k},
$$

where

$$
C_{5}\left(\varepsilon_{5}\right)=\frac{(\sqrt{s})^{2 s+1} C_{4}^{2 s-1}(\varepsilon) e^{2(2 s-1) \varepsilon k} C_{3}^{2 s}(\varepsilon)}{\left(\sqrt{\frac{1}{t K}}\right)^{2 s}} C^{3 s+2 s K-1}(\varepsilon)
$$

and

$$
\varepsilon_{5}=\varepsilon(9 s+2 s K-3) .
$$

Combining (57) with (58) and (60), we obtain

$$
\begin{aligned}
\|U(k)\| & \leq\left(\gamma^{2}(k)+1\right) \beta_{1}\left(\gamma^{2}(k)+1, \gamma^{-2 s}(k),(k-1) K\right) \\
& \leq\left(C_{3}^{2}(\varepsilon) e^{2 \varepsilon k}+1\right) C_{5}\left(\varepsilon_{5}\right) e^{\varepsilon_{5} k}
\end{aligned}
$$

It is clear that there exists a constant $C_{6}(\varepsilon)>0$ such that

$$
C_{3}^{2}(\varepsilon) s e^{2 \varepsilon k}+s+1 \leq C_{6}(\varepsilon) e^{2 \varepsilon k}
$$


and therefore,

$$
\|U(k)\| \leq C_{6}(\varepsilon) C_{5}\left(\varepsilon_{5}\right) e^{\left(2 \varepsilon+\varepsilon_{5}\right) k}
$$

The last inequality implies

$$
\limsup _{k \rightarrow \infty} \frac{1}{k} \ln \|U(k)\|=0,
$$

since $\varepsilon_{5}$ tends to zero when $\varepsilon$ does so.

Definition 7 (Global positive scalarizability) We say that the system (7) is globally positively scalarizable if for any sequence $p=(p(k))_{k \in \mathbb{N}}, p^{-1} \in \mathcal{L}^{\text {tem }}(\mathbb{N}, \mathbb{R})$, of positive real numbers, there exists an admissible control $U=(U(k))_{k \in \mathbb{N}}$ such that (7) is weakly equivalent to the system (1) with $A(k)=p(k) I_{s}$.

Theorem 11 (Sufficient condition for global positive scalarizability) Suppose that $A, A^{-1} \in$ $\mathcal{L}^{\text {tem }}\left(\mathbb{N}, \mathbb{R}^{s \times s}\right), B \in \mathcal{L}^{\text {tem }}\left(\mathbb{N}, \mathbb{R}^{s \times t}\right)$ and system (5) is $K$-completely controllable and

$$
\liminf _{k \rightarrow \infty} \frac{1}{k} \ln \alpha_{A, B}((k-1) K, k K) \geq 0 .
$$

Then system (7) is globally positively scalarizable.

Proof Assume that system (5) is $K$-completely controllable. Let $T=(T(k))_{k \in \mathbb{N}}$ be a sequence according to Corollary 1 . By the QR factorization theorem (see [49, p. 112]) for the invertible matrix $T^{-1}(k+1) \Phi_{A}(k K,(k-1) K) T(k)$, there is an orthogonal matrix $Q(k)$ and an upper triangular matrix $R(k)$ with positive diagonal elements such that

$$
T^{-1}(k+1) \Phi_{A}(k K,(k-1) K) T(k)=Q(k) R(k),
$$

and consequently,

$$
\Phi_{A}(k K,(k-1) K) T(k) R^{-1}(k) T^{-1}(k)=T(k+1) Q(k) T^{-1}(k) .
$$

Since

$$
R(k)=Q^{-1}(k) T^{-1}(k+1) \Phi_{A}(k K,(k-1) K) T(k),
$$

it follows that $R=(R(k))_{k \in \mathbb{N}}$ is a tempered sequence. Consider any positive sequence $p=$ $(p(k))_{k \in \mathbb{N}}$ such that $p, p^{-1} \in \mathcal{L}^{\text {tem }}(\mathbb{N}, \mathbb{R})$ and denote

$$
\begin{aligned}
& \varphi(k, m)=\prod_{i=m}^{k-1} p(i) \text { for } k>m, \\
& \varphi(k, k)=1
\end{aligned}
$$

and

$$
\varphi(m, k)=\varphi^{-1}(k, m) \quad \text { for } k>m .
$$


Note that

$$
\varphi(k, m)=\varphi(k, k) \varphi(k, m)
$$

for all $k, m \in \mathbb{N}$.

Let us fix $\varepsilon>0$. Since $A, A^{-1}, B, T, T^{-1}, R, R^{-1}, p$ and $p^{-1}$ are tempered sequences, there exists a constant $C(\varepsilon)>0$ such that

$$
\max \left\{a((k-1) K, K),\|R(k)\|,\left\|T^{-1}(k)\right\|,\|T(k)\|, \max _{i=0, \ldots, K-1} \varphi(k K, k K-i)\right\} \leq C(\varepsilon) e^{\varepsilon k}
$$

For an upper triangular matrix

$$
\bar{H}(k)=R^{-1}(k) \varphi(k K,(k-1) K)
$$

with positive diagonal elements, we have

$$
\begin{aligned}
\|\bar{H}(k)\|= & \left\|R^{-1}(k)\right\| \varphi(k K,(k-1) K) \\
\leq & \|Q(k)\|\|T(k+1)\|\left\|\Phi_{A}^{-1}(k K,(k-1) K)\right\| \\
& \times\left\|T^{-1}(k)\right\| \varphi(k K,(k-1) K) \\
\leq & C^{4}(\varepsilon) e^{4 \varepsilon k} \\
\left\|\bar{H}^{-1}(k)\right\|= & \|R(k)\| \varphi((k-1) K, k K) \leq C^{4}(\varepsilon) e^{4 \varepsilon k} ;
\end{aligned}
$$

therefore, $(\bar{H}(k))_{k \in \mathbb{N}}$ is a tempered sequence. According to the definition of $T(k)$ and Corollary 1, we know that there exists a feedback control $U=(U(i))_{i \in \mathbb{N}}$ such that

$$
\begin{aligned}
\Phi_{A+B U}(k K,(k-1) K) & =\Phi_{A}(k K,(k-1) K) T(k) \bar{H}(k) T^{-1}(k) \\
& =\Phi_{A}(k K,(k-1) K) T(k) R^{-1}(k) T^{-1}(k) \varphi(k K,(k-1) K) \\
& =T(k+1) Q(k) T^{-1}(k) \varphi(k K,(k-1) K), \quad k \in \mathbb{N} .
\end{aligned}
$$

Multiplying these equalities, we get

$$
\Phi_{A+B U}(k K, 0)=T(k+1) \widetilde{Q}(k) T^{-1}(1) \varphi(k K, 0), \quad k \in \mathbb{N},
$$

where

$$
\widetilde{Q}(k)=Q(k) Q(k-1) \cdots Q(1)
$$

Note that $\widetilde{Q}(k)$ is orthogonal as a product of orthogonal matrices. As it follows from Corollary 1, the sequences $(A(k)+B(k) U(k))_{k \in \mathbb{N}}$ and $\left((A(k)+B(k) U(k))^{-1}\right)_{k \in \mathbb{N}}$ are tempered and (61) holds.

Let us define a sequence $D=(D(i))_{i \in \mathbb{N}}$ as follows

$$
D(i)=\Phi_{A+B U}(i, 0) \varphi(0, i), \quad i \in \mathbb{N}
$$


We will show that $D$ is a tempered sequence. Let us fix $i \in \mathbb{N}$. Then there exists exactly one $k \in \mathbb{N}$ such that $i \in[k K,(k+1) K)$. By the properties of the transition matrix and the function $\varphi(m, k)$, we get

$$
\begin{aligned}
D(i) & =\Phi_{A+B U}(i, 0) \varphi(0, i) \\
& =\Phi_{A+B U}(i, k K) \Phi_{A+B U}(k K, 0) \varphi(0, k K) \varphi(k K, i) \\
& =\Phi_{A+B U}(i, k K) T(k+1) \widetilde{Q}(k) T^{-1}(1) \varphi(k K, i) .
\end{aligned}
$$

Since $i-k K<K$, it follows that

$$
\begin{aligned}
\|D(i)\| & \leq\left\|\Phi_{A+B U}(i, k K)\right\|\|T(k+1)\|\|\widetilde{Q}(k)\|\left\|T^{-1}(1)\right\| \varphi(k K, i) \\
& \leq C^{K+3}(\varepsilon) e^{K \varepsilon k+3 \varepsilon k}
\end{aligned}
$$

and similarly

$$
\left\|D^{-1}(i)\right\| \leq C^{K+3}(\varepsilon) e^{K \varepsilon k+3 \varepsilon k}
$$

Observe that the sequence $D$ establishes weak equivalence of the system (7) with the defined control $U$ and system (1) with

$$
C(k)=p(k) I_{s}
$$

In fact,

$$
\begin{aligned}
& D^{-1}(i+1)(A(i)+B(i) U(i)) D(i) \\
& \quad=\varphi(i+1,0) \Phi_{A+B U}(0, i+1)(A(i)+B(i) U(i)) \Phi_{A+B U}(i, 0) \varphi(0, i) \\
& \quad=\varphi(i+1, i) \Phi_{A+B U}(0, i+1) \Phi_{A+B U}(i+1,0) \\
& \quad=\varphi(i+1, i) I_{s}=p(i) I_{s}, \quad i \in \mathbb{N} .
\end{aligned}
$$

From Theorem 6, we obtain the following corollary.

Corollary 2 Under the assumption of Theorem 11, there exists an admissible feedback control $U_{1}=\left(U_{1}(k)\right)_{k \in \mathbb{N}}$ such that $(7)$ is weakly equivalent to

$$
y(k+1)=y(k)
$$

In our further considerations, we will use the following result.

Lemma 7 Suppose that $A=(A(k))_{k \in \mathbb{N}}, C=(C(k))_{k \in \mathbb{N}}$ and $A^{-1}, C^{-1}$ are tempered sequences and assume that

$$
\Phi_{C}\left(k_{k+1}, k_{k}\right)=\Phi_{A}\left(k_{k+1}, k_{k}\right)
$$


for all $k \in \mathbb{N}$, where $\left(k_{k}\right)_{k \in \mathbb{N}}$ is a sequence of natural numbers such that $0<k_{k+1}-k_{k} \leq c<\infty$ for all $k \in \mathbb{N}$. Then the systems (1) and

$$
x(k+1)=C(k) x(k)
$$

are weakly equivalent.

Proof Let us fix $\varepsilon>0$ and consider $M(\varepsilon)>1$ such that

$$
\|A(k)\|+\left\|A^{-1}(k)\right\|+\|C(k)\|+\left\|C^{-1}(k)\right\| \leq M(\varepsilon) e^{\varepsilon k} \quad \text { for all } k \in \mathbb{N}
$$

and define

$$
D(k)=\Phi_{C}\left(k, k_{1}\right) \Phi_{A}\left(k_{1}, k\right), \quad k \in \mathbb{N}
$$

Let us fix $k \in \mathbb{N}$. Then there exists a unique $k \in \mathbb{N}$ such that $k_{k} \leq k<k_{k+1}$. Observe that $0 \leq k-k_{k} \leq c$. Moreover,

$$
\begin{aligned}
D(k) & =\Phi_{C}\left(k, k_{k}\right) \Phi_{C}\left(k_{k}, k_{1}\right) \Phi_{A}\left(k_{1}, k_{k}\right) \Phi_{A}\left(k_{k}, k\right) \\
& =\Phi_{C}\left(k, k_{k}\right) \Phi_{C}\left(k_{k}, k_{k-1}\right) \cdots \Phi_{C}\left(k_{2}, k_{1}\right) \Phi_{A}\left(k_{1}, k_{2}\right) \cdots \Phi_{A}\left(k_{k-1}, k_{k}\right) \Phi_{A}\left(k_{k}, k\right) \\
& =\Phi_{C}\left(k, k_{k}\right) \Phi_{A}\left(k_{k}, k\right)
\end{aligned}
$$

and

$$
D^{-1}(k)=\Phi_{A}^{-1}\left(k_{k}, k\right) \Phi_{C}^{-1}\left(k, k_{k}\right)=\Phi_{A}\left(k, k_{k}\right) \Phi_{C}\left(k_{k}, k\right) .
$$

From the last two equalities we get

$$
\begin{aligned}
& \|D(k)\| \leq M^{2\left|k-k_{k}\right|}(\varepsilon) e^{\varepsilon k 2\left|k-k_{k}\right|} \leq M^{2 c}(\varepsilon) e^{2 c \varepsilon k} \\
& \left\|D^{-1}(k)\right\| \leq M^{2\left|k-k_{k}\right|}(\varepsilon) e^{\varepsilon k 2\left|k-k_{k}\right|} \leq M^{2 c}(\varepsilon) e^{2 c \varepsilon k}
\end{aligned}
$$

This implies that the sequence $(D(k))_{k \in \mathbb{N}}$ is a tempered sequence. Finally,

$$
\begin{aligned}
D^{-1}(k+1) C(k) D(k) & =\Phi_{A}\left(k+1, k_{1}\right) \Phi_{C}\left(k_{1}, k+1\right) C(k) \Phi_{C}\left(k, k_{1}\right) \Phi_{A}\left(k_{1}, k\right) \\
& =\Phi_{A}\left(k+1, k_{1}\right) \Phi_{C}\left(k_{1}, k+1\right) \Phi_{C}\left(k+1, k_{1}\right) \Phi_{A}\left(k_{1}, k\right) \\
& =\Phi_{A}\left(k+1, k_{1}\right) \Phi_{A}\left(k_{1}, k\right)=A(k) .
\end{aligned}
$$

The following theorem plays a key role in obtaining our main results.

Theorem 12 Suppose that $A, A^{-1} \in \mathcal{L}^{\text {tem }}\left(\mathbb{N}, \mathbb{R}^{s \times s}\right), B \in \mathcal{L}^{\text {tem }}\left(\mathbb{N}, \mathbb{R}^{s \times t}\right)$ and system (5) is $K$ completely controllable and

$$
\liminf _{k \rightarrow \infty} \frac{1}{k} \ln \alpha_{A, B}((k-1) K, k K) \geq 0 .
$$


Then for any positive sequences $p_{i}=\left(p_{i}(k)\right)_{k \in \mathbb{N}}, p_{i}^{-1} \in \mathcal{L}^{\text {tem }}(\mathbb{N}, \mathbb{R}), i=1, \ldots, s$, there exists an admissible feedback control $U=(U(k))_{k \in \mathbb{N}}$ such that system (7) is weakly dynamically equivalent to

$$
z(k+1)=C(k) z(k)
$$

where $(C(k))_{k \in \mathbb{N}}$ is an upper triangular tempered sequence with $p_{i}(k), i=1, \ldots, s$, on the main diagonal for all $k \in \mathbb{N}$.

Proof Consider positive sequences $p_{i}=\left(p_{i}(k)\right)_{k \in \mathbb{N}}, p_{i}^{-1} \in \mathcal{L}^{\text {tem }}(\mathbb{N}, \mathbb{R}), i=1, \ldots, s$, and denote

$$
\gamma(k)=\max _{i=1, \ldots, s}\left\{\sup _{l=0, \ldots, k} p_{i}(l), \sup _{l=0, \ldots, k} p_{i}^{-1}(l)\right\}
$$

According to Corollary 2, there exists a feedback control $U_{1}=\left(U_{1}(k)\right)_{k \in \mathbb{N}}$ such that

$$
\limsup _{k \rightarrow \infty} \frac{1}{k} \ln \left\|U_{1}(k)\right\|=0
$$

and the system

$$
x(k+1)=\left(A(k)+B(k) U_{1}(k)\right) x(k), \quad k \in \mathbb{N}
$$

is weakly dynamically equivalent to

$$
y(k+1)=y(k), \quad k \in \mathbb{N} .
$$

Let

$$
y(k)=D(k) x(k)
$$

be the transformation that establishes this equivalence. Then we have

$$
I_{s}=D(k+1)\left(A(k)+B(k) U_{1}(k)\right) D^{-1}(k), \quad k \in \mathbb{N},
$$

and consequently,

$$
D^{-1}(k+1) D(k)=A(k)+B(k) U_{1}(k), \quad k \in \mathbb{N} .
$$

Note that this sequence establishes also a weak dynamic equivalence of

$$
x(k+1)=\left(A(k)+B(k) U_{1}(k)\right) x(k)+B(k) u(k)
$$

and

$$
y(k+1)=y(k)+D(k+1) B(k) u(k) .
$$


By Lemma 3, we know that (66) is $K$-completely controllable. Since by Lemma 4 , dynamic equivalence preserves $K$-complete controllability, it follows that system (66) is $K$ completely controllable and satisfies (63).

According to Corollary 1 , there exists a sequence $T=(T(k))_{k \in \mathbb{N}}, T, T^{-1} \in \mathcal{L}^{\text {tem }}\left(\mathbb{N}, \mathbb{R}^{s \times s}\right)$ such that for each upper triangular sequence $(L(k))_{k \in \mathbb{N}},\left(L^{-1}(k)\right)_{k \in \mathbb{N}} \in \mathcal{L}^{\text {tem }}\left(\mathbb{N}, \mathbb{R}^{s \times s}\right), L(k) \in$ $\mathrm{GL}_{s}(\mathbb{R})$ and each lower triangular sequence $(G(k))_{k \in \mathbb{N}},\left(G^{-1}(k)\right)_{k \in \mathbb{N}} \in \mathcal{L}^{\text {tem }}\left(\mathbb{N}, \mathbb{R}^{s \times s}\right)$ of matrices with positive diagonal elements (both $L$ and $G$ ), there exists a control $V=(V(k))_{k \in \mathbb{N}}$ such that

$$
\limsup _{k \rightarrow \infty} \frac{1}{k} \ln \|V(k)\|=0
$$

and

$$
Y_{V}(k K,(k-1) K)=T(k) L(k) G(k) T^{-1}(k),
$$

where $Y_{V}$ is the transition matrix of

$$
y(k+1)=\left(I_{s}+D(k+1) B(k) V(k)\right) y(k) .
$$

Denote

$$
f(k)=\max \left\{\sup _{i=0, \ldots, k}\|T(i)\|, \sup _{i=0, \ldots, k}\left\|T^{-1}(i)\right\|\right\}
$$

Let us apply the QR factorization theorem to $T(k)$ and let

$$
T(k)=R(k) Q(k),
$$

where $R(k)$ and $Q(k)$ are the upper triangular and orthogonal matrices, respectively. We have

$$
\|R(k)\|=\left\|T(k) Q^{-1}(k)\right\| \leq f(k)
$$

and

$$
\left\|R^{-1}(k)\right\|=\left\|Q(k) T^{-1}(k)\right\| \leq f(k) .
$$

Denote

$$
J(k)=\operatorname{diag}\left(\prod_{j=(k-1) K}^{k K-1} \sqrt{p_{1}(j)}, \ldots, \prod_{j=(k-1) K}^{k K-1} \sqrt{p_{s}(j)}\right)
$$

Applying the QR factorization theorem to $J(k) Q(k)$, we have

$$
J(k) Q(k)=\widetilde{Q}(k) \widetilde{R}(k),
$$


where $\widetilde{R}(k)$ is an upper triangular matrix, and $\widetilde{Q}(k)$ is an orthogonal matrix. Moreover,

$$
\|\widetilde{R}(k)\| \leq\left\|\widetilde{Q}^{-1}(k) J(k) Q(k)\right\| \leq\|J(k)\|=\gamma^{K / 2}(k)
$$

and similarly,

$$
\left\|\widetilde{R}^{-1}(k)\right\| \leq \gamma^{K / 2}(k)
$$

Corollary 1 implies that for the control $V=(V(k))_{k \in \mathbb{N}}$, we have

$$
\begin{aligned}
\Phi_{V} & (k K,(k-1) K) \\
& =T(k)(\widetilde{R}(k))^{T} \widetilde{R}(k) T^{-1}(k) \\
& =T(k)(\widetilde{R}(k))^{T}(\widetilde{Q}(k))^{T} \widetilde{Q}(k) \widetilde{R}(k) T^{-1}(k) \\
& =T(k) Q^{T}(k) J^{T}(k) J(k) Q(k) T^{-1}(k) \\
& =T(k) Q^{T}(k) J^{2}(k) Q(k) T^{-1}(k) \\
& =R(k) Q(k) Q^{T}(k) J^{2}(k) Q(k) Q^{-1}(k) R^{-1}(k) \\
& =R(k) J^{2}(k) R^{-1}(k) \\
& =R(k) \operatorname{diag}\left(\prod_{j=(k-1) K}^{k K-1} p_{1}(j), \ldots, \prod_{j=(k-1) K}^{k K-1} p_{s}(j)\right) R^{-1}(k) .
\end{aligned}
$$

Consider the system (64) with

$$
C(k)=R(l) \operatorname{diag}\left(p_{1}(k), \ldots, p_{s}(k)\right) R^{-1}(l)
$$

for

$$
k \in[(l-1) K, l K) .
$$

The matrices $C(k)$ are upper triangular with $p_{i}(k), i=1, \ldots, s$, on the main diagonal and

$$
\begin{aligned}
& \|C(k)\| \leq\|R(l)\|\left\|\operatorname{diag}\left(p_{1}(k), \ldots, p_{s}(k)\right)\right\|\left\|R^{-1}(l)\right\| \leq f(k)^{2} \gamma(k), \quad k \in \mathbb{N}, \\
& \left\|C^{-1}(k)\right\| \leq\left\|R^{-1}(l)\right\|\left\|\operatorname{diag}\left(p_{1}^{-1}(k), \ldots, p_{s}^{-1}(k)\right)\right\|\|R(l)\| \leq f^{2}(k) \gamma(k), \quad k \in \mathbb{N} .
\end{aligned}
$$

This implies that $(C(k))_{k \in \mathbb{N}}$ and $\left(C^{-1}(k)\right)_{k \in \mathbb{N}}$ are tempered sequences.

For the transition matrix $\Phi_{C}$ of (64), we have

$$
\begin{aligned}
\Phi_{C}(l K,(l-1) K) & =C(l K-1) \cdots C((l-1) K) \\
& =R(l) \operatorname{diag}\left(\prod_{j=(l-1) K}^{l K-1} p_{1}(j), \ldots, \prod_{j=(l-1) K}^{l K-1} p_{s}(j)\right) R^{-1}(l) \\
& =\Phi_{V}(l K,(l-1) K), \quad l \in \mathbb{N} .
\end{aligned}
$$


According to Lemma 7, the system (69) with the defined control $V$ and the system (64) are dynamically equivalent. Applying to (69) the inverse Lyapunov transformation

$$
x(k)=D^{-1}(k) y(k)
$$

we get

$$
\begin{aligned}
x(k+1) & =D^{-1}(k+1) y(k+1) \\
& =D^{-1}(k+1)\left(I_{s}+D(k+1) B(k) V(k)\right) D(k) x(k) \\
& =\left(D^{-1}(k+1) D(k)+B(k) V(k) D(k)\right) x(k) \\
& =\left(A(k)+B(k) U_{1}(k)+B(k) V(k) D(k)\right) x(k) \\
& =(A(k)+B(k) U(k)) x(k),
\end{aligned}
$$

where

$$
U(k)=U_{1}(k)+V(k) D(k), \quad k \in \mathbb{N} .
$$

We see that the system (7) with the defined control $U$ is dynamically equivalent to (64). The fact that $(A(k)+B(k) U(k))_{k \in \mathbb{N}}$ and $\left((A(k)+B(k) U(k))^{-1}\right)_{k \in \mathbb{N}}$ are tempered follows from the fact that $(C(k))_{k \in \mathbb{N}}$ and $\left(C^{-1}(k)\right)_{k \in \mathbb{N}}$ are tempered sequences and

$$
A(k)+B(k) U(k)=D_{1}^{-1}(k+1) C(k) D_{1}(k), \quad k \in \mathbb{N},
$$

where $\left(D_{1}(k)\right)_{k \in \mathbb{N}}$ is the tempered sequence that establishes the dynamic equivalence of (7) and (64). Finally, (8) follows from (65), (68), (70), and the fact that $(D(k))_{k \in \mathbb{N}}$ and $\left(D^{-1}(k)\right)_{k \in \mathbb{N}}$ are tempered.

\section{Proof of Theorem 3}

We will start with some facts about NEDS of one-dimensional systems.

Remark 7 Observe that for one-dimensional systems, the definition of NED means that there exist constants $\alpha, \eta_{0}>0$ such that for all $\varepsilon \in\left(0, \eta_{0}\right)$, there exists $K(\varepsilon)>0$ such that either

$$
\prod_{i=k}^{l-1}|c(i)| \leq K(\varepsilon) e^{-\alpha(l-k)+\varepsilon k}, \quad k, l \in \mathbb{N}, l \geq k,
$$

or

$$
\prod_{i=l}^{k-1}|c(i)| \geq \frac{1}{K(\varepsilon)} e^{\alpha(k-l)-\varepsilon k}, \quad k, l \in \mathbb{N}, l \leq k .
$$

Remark 8 In particular, if a one-dimensional sequence $(c(n))_{n \in \mathbb{N}}$ has a NED, then either the Lyapunov exponent

$$
\lambda(c):=\limsup _{n \rightarrow \infty} \frac{1}{n} \sum_{i=1}^{n} \ln |c(i)|
$$


is negative and satisfies $\lambda(c) \leq-\alpha<0$, or the Perron exponent

$$
\underline{\lambda}(c):=\liminf _{n \rightarrow \infty} \frac{1}{n} \sum_{i=1}^{n} \ln |c(i)|
$$

is positive and satisfies $\underline{\lambda}(c)>\alpha>0$.

The next lemma shows that for one-dimensional systems the NEDS may have all the forms described in Theorem 1 .

Lemma 8 Let $\bar{\Sigma}$ be one of the sets $\emptyset, \mathbb{R},[x, \infty),(-\infty, x]$ or $[x, y]$ where $x<y$. Then there exists a scalar sequence a such that the NEDS $\Sigma(a)$ of a equals $\bar{\Sigma}$.

Proof Consider first the case $\bar{\Sigma}=[x, y]$. Let us take any sequence $b=(b(n))_{k \in \mathbb{N}}$ such that $b(n) \in[x, y]$ and

$$
\limsup _{n \rightarrow \infty} \frac{1}{n} \sum_{i=1}^{n} b(i)=y
$$

and

$$
\liminf _{n \rightarrow \infty} \frac{1}{n} \sum_{i=1}^{n} b(i)=x
$$

and define the sequence $a=(a(n))_{k \in \mathbb{N}}$ by

$$
a(n)=\exp (b(n)+\sqrt{n+1} \cos (n+1)-\sqrt{n} \cos n) .
$$

Observe that from the construction, it follows that we have

$$
\Phi_{a}(m, n)=\exp \left(\sum_{i=n}^{m-1} b(i)+\sqrt{m} \cos m-\sqrt{n} \cos n\right) \quad \text { for } m \geq n .
$$

We will show that $\Sigma(a)=[x, y]$. We will do this in three steps.

Step 1. For each $\gamma>y$, the system $c=\left(e^{-\gamma} a(n)\right)_{k \in \mathbb{N}}$ satisfies condition (71). Since $b(n) \leq y$,

$$
\begin{aligned}
\Phi_{c}(m, n) & =\exp \left(\sum_{i=n}^{m-1}(b(i)-\gamma)+\sqrt{m} \cos m-\sqrt{n} \cos n\right) \\
& \leq \exp \left(\sum_{i=n}^{m-1}(y-\gamma)+\sqrt{m} \cos m-\sqrt{n} \cos n\right) \\
& \leq \exp ((y-\gamma)(m-n)+\sqrt{m}+\sqrt{n})
\end{aligned}
$$

for $m \geq n$. Let us fix $\varepsilon>0$. Since $\sqrt{n}-\frac{\varepsilon}{2} n \rightarrow-\infty$, when $n \rightarrow \infty$, there exists $D(\varepsilon)>0$ such that

$$
e^{\sqrt{n}} \leq D(\varepsilon) e^{\frac{\varepsilon}{2} n} \quad \text { for } n \in \mathbb{N} \text {. }
$$


Hence,

$$
\begin{aligned}
\Phi_{c}(m, n) & \leq \exp ((y-\gamma)(m-n)+\sqrt{m}+\sqrt{n}) \\
& \leq D^{2}(\varepsilon) \exp \left((y-\gamma)(m-n)+\frac{\varepsilon}{2} m+\frac{\varepsilon}{2} n\right) \\
& =D^{2}(\varepsilon) \exp \left((y-\gamma)(m-n)+\frac{\varepsilon}{2}(m-n)+\varepsilon n\right) \\
& =D^{2}(\varepsilon) \exp \left(-\left(-y+\gamma-\frac{\varepsilon}{2}\right)(m-n)+\varepsilon n\right) \\
& \leq K(\varepsilon) e^{-\alpha(m-n)+\varepsilon n},
\end{aligned}
$$

where $K(\varepsilon)=D^{2}(\varepsilon), \alpha=-y+\gamma$, provided that $\varepsilon \leq 4 \gamma$. This proves (71), see Remark 7 .

Step 2. For each $\gamma<x$, the system $c=\left(e^{-\gamma} a(n)\right)_{k \in \mathbb{N}}$ satisfies condition (72). Since $b(n) \geq x$,

$$
\begin{aligned}
\Phi_{c}(m, n) & =\exp \left(\sum_{i=n}^{m-1}(b(i)-\gamma)+\sqrt{m} \cos m-\sqrt{n} \cos n\right) \\
& \geq \exp \left(\sum_{i=n}^{m-1}(x-\gamma)+\sqrt{m} \cos m-\sqrt{n} \cos n\right) \\
& \geq \exp ((x-\gamma)(m-n)-\sqrt{m}-\sqrt{n}) .
\end{aligned}
$$

For a fixed $\varepsilon>0$, we conclude from (75) that

$$
\begin{aligned}
\Phi_{c}(m, n) & \geq \exp ((x-\gamma)(m-n)-\sqrt{m}-\sqrt{n}) \\
& \geq D^{-2}(\varepsilon) \exp \left((x-\gamma)(m-n)-\frac{\varepsilon}{2} m-\frac{\varepsilon}{2} n\right) \\
& =D^{-2}(\varepsilon) \exp \left((x-\gamma)(m-n)-\frac{\varepsilon}{2}(m-n)-\varepsilon n\right) \\
& =D^{-2}(\varepsilon) \exp \left(-\left(-x+\gamma+\frac{\varepsilon}{2}\right)(m-n)-\varepsilon n\right) \\
& \geq \frac{1}{K(\varepsilon)} e^{\alpha(m-n)-\varepsilon n},
\end{aligned}
$$

where $K(\varepsilon)=D^{2}(\varepsilon), \alpha=x-\gamma$, provided that $\varepsilon \leq 4 \gamma$. This proves (72), see Remark 7 .

Step 3. For each $\gamma \in[x, y]$, the system $c=\left(e^{-\gamma} a(n)\right)_{k \in \mathbb{N}}$ does not have a NED. Indeed, from (74) and (73), we get

$$
\lambda(c)=y-\gamma>0
$$

and

$$
\underline{\lambda}(c)=x-\gamma<0 \text {. }
$$

In a very similar manner, we may construct a sequence $a$ such that $\Sigma(a)$ is $\mathbb{R}$ or $(-\infty, x]$ or $[x, \infty)$. Finally, it is easy to check that for $a(n)=n+1$, we have $\Sigma(a)=\emptyset$. 
Proof of Theorem 3 Let $\bar{\Sigma}=\emptyset, \bar{\Sigma}=\mathbb{R}$ or $\bar{\Sigma}=\bigcup_{i=1}^{q} I_{i}$, where $I_{1}=\left[a_{1}, b_{1}\right]$ or $I_{1}=\left(-\infty, b_{1}\right]$, $I_{i}=\left[a_{i}, b_{i}\right], i=2, \ldots, q-1$, and $I_{q}=\left[a_{q}, b_{q}\right]$ or $I_{q}=\left[a_{q},+\infty\right)$ for some numbers $a_{1} \leq b_{1}<$ $a_{2} \leq b_{2}<\cdots<a_{q} \leq b_{q}$ and $q \leq s$. Consider now the case when $\bar{\Sigma}=\bigcup_{i=1}^{q} I_{i}$. For $1 \leq i \leq q$, we construct a sequence $p_{i}=\left(p_{i}(k)\right)_{k \in \mathbb{N}}, p_{i}^{-1} \in \mathcal{L}^{\text {tem }}(\mathbb{N}, \mathbb{R})$ of positive real numbers such that $\Sigma\left(p^{i}\right)=I_{i}$. This is possible by Lemma 8 . For $q+1 \leq i \leq s$, let $p_{i}(k)=p_{1}(k)$. According to Theorem 12, there exists an admissible feedback control $(U(k))_{k \in \mathbb{N}}$ and a sequence of upper triangular matrices $(C(k))_{k \in \mathbb{N}},\left(C^{-1}(k)\right)_{k \in \mathbb{N}} \in \mathcal{L}^{\text {tem }}\left(\mathbb{N}, \mathbb{R}^{n \times n}\right)$, where $C(k)=\left(c_{i j}(k)\right)_{1 \leq i, j \leq n}$ with $c_{i i}(k)=p_{i}(k)$ such that

$$
x(k+1)=(A(k)+B(k) U(k)) x(k), \quad y(k+1)=C(k) y(k)
$$

are weakly equivalent. From the definition of weak equivalence, it is clear that weakly equivalent systems have the same NEDS; therefore, using Theorem 7 , we get

$$
\Sigma(A+B U)=\Sigma(C)=\bigcup_{i=1}^{s} \Sigma\left(p_{i}\right)=\bigcup_{i=1}^{q}\left[a_{i}, b_{i}\right]
$$

In the case of $\bar{\Sigma}$ being $\emptyset$ or $\mathbb{R}$, the proof is analogical. We put $p_{i}(k)=p(k)$ for all $1 \leq i \leq$ $s$, where $(p(k))_{k \in \mathbb{N}}, p^{-1} \in \mathcal{L}^{\text {tem }}(\mathbb{N}, \mathbb{R})$ is any sequence of positive real numbers such that $\Sigma(p)=\emptyset$ or $\Sigma(p)=\mathbb{R}$.

\section{Example}

Example 1 Let us consider the sequence $a=(a(n))_{n \in \mathbb{N}}$ from the proof of Lemma 8 for $x=2$ and $y=3$. Consider the uncontrolled system (1) with

$$
A(k)=\frac{1}{2}\left[\begin{array}{cc}
1+a(k) & 1-a(k) \\
1-a(k) & 1+a(k)
\end{array}\right] \text { for } k \in \mathbb{N} .
$$

For this system we have $\Sigma(A)=\{1,[2,3]\}$ and in particular, the system is not stable. It is also clear that $A, A^{-1} \in \mathcal{L}^{\text {tem }}\left(\mathbb{N}, \mathbb{R}^{2 \times 2}\right)$. Consider now the controlled system (5) with

$$
B(k)=\frac{1}{2}\left[\begin{array}{cc}
\frac{\sqrt{2}}{k+1} & -\sqrt{2} \\
\frac{\sqrt{2}}{k+1} & \sqrt{2}
\end{array}\right] .
$$

It is clear that $B \in \mathcal{L}^{\text {tem }}\left(\mathbb{N}, \mathbb{R}^{2 \times 2}\right)$ and for $l>k$, we have

$$
W_{A, B}(k, l)=\frac{1}{2}\left[\begin{array}{ll}
\lambda(k, l)+\mu(k, l) & \lambda(k, l)-\mu(k, l) \\
\lambda(k, l)-\mu(k, l) & \lambda(k, l)+\mu(k, l)
\end{array}\right],
$$

where

$$
\lambda(k, l)=\sum_{j=k}^{l-1} \frac{1}{(j+1)^{2}}, \quad \mu(k, l)=\sum_{j=k}^{l-1} \exp \left(2 \sum_{i=k}^{j} b(i)+2 \sqrt{j+1} \cos (j+1)-2 \sqrt{k} \cos k\right) .
$$

Moreover, the numbers $\lambda(k, l)>0$ and $\mu(k, l)>0$ are the eigenvalues of $W_{A, B}(k, l)$ and satisfy

$$
\lim _{k \rightarrow \infty} \frac{1}{k} \ln \lambda(k, k+1)=\lim _{k \rightarrow \infty} \frac{1}{k} \ln \mu(k, k+1)=0 .
$$


Therefore, the assumption of Theorem 3 is satisfied, and the considered system has the NEDS assignable. If we want to stabilize the system, we may try to construct an admissible feedback $U$ such that $\Sigma(A+B U)=\{-1\}$. In fact, if we define an admissible feedback as

$$
U(k)=\frac{1}{2} \sqrt{2}\left[\begin{array}{cc}
-2 k-2 & -2 k-2 \\
a(k)+1 & -a(k)-1
\end{array}\right],
$$

then

$$
A(k)+B(k) U(k)=\left[\begin{array}{cc}
-1 & 0 \\
0 & -1
\end{array}\right] .
$$

Observe also that the coefficients of the controlled system (5) considered in this example are unbounded, and therefore, the result of papers [30-34] can not be applied here to stabilize this system.

\section{Conclusion}

In this paper, we investigated a problem of assignability of nonuniform dichotomy spectrum by time-varying linear feedback for linear discrete time-varying systems with tempered coefficients. The main result is that the spectrum is assignable if the system is completely controllable and certain lower asymptotic bound for the controllability Gramian holds (see (9)). To obtain this result, we generalize to the case of tempered and completely controllable systems the Theorem 4.6 from [30], which makes it possible to bring the system into an upper triangular form through linear feedback. The original theorem was proved for uniformly completely controllable systems with bounded coefficients. To the upper triangular system, we apply the idea of linking operator proposed by Batelli and Palmer in [39], and we obtain the result that the nonuniform dichotomy spectrum of an upper triangular system is the union of spectra of the one-dimensional systems from its main diagonal. It has been recently shown (see [33, 34], and [35]) that for systems with bounded coefficients, the assignability of uniform exponential dichotomy spectrum is equivalent to uniform complete controllability. It is an open question whether our sufficient conditions for assignability of the nonuniform dichotomy spectrum of systems with tempered coefficients are also necessary.



\section{Declarations}

Competing interests

The authors declare that they have no competing interests. 


\section{Author details}

'Department of Automatic Control and Robotics, Silesian University of Technology, Gliwice, Poland. ${ }^{2}$ Faculty of Mathematics, Technische Universität Dresden, 01062 Dresden, Germany.

\section{Publisher's Note}

Springer Nature remains neutral with regard to jurisdictional claims in published maps and institutional affiliations.

Received: 11 April 2021 Accepted: 11 December 2021 Published online: 04 March 2022

\section{References}

1. Lyapunov, A.M.: General Problem of Stability of Motion, Collected Works, vol. 2. Izdat. Akad. Nauk SSSR, Moscow (1956) (in Russian)

2. Perron, O.: Die Stabilitätsfrage bei Differentialgleichungen. Math. Z. 32, 703-728 (1930) (in German)

3. Maizel, A.D.: Ob ustoichivosti resheniy sistem differentsialnyh uravneniy. Tr. Politehnich. Inst. 51, $20-50$ (1954) (in Russian)

4. Persidsky, K.: Über die Stabilität einer Bewegung nach der ersten Näherung. Mat. Sb. 40, 284-293 (1933)

5. Malkin, I.: Über die Stabilität der Bewegung im Sinne von Liapounoff. Mat. Sb. 45, 47-101 (1938)

6. Hadamard, J.: Sur l'itération et les solutions asymptotiques des équations différentielles. Bull. Soc. Math. Fr. 29, 224-228 (1901) (in French)

7. Bohl, P.: Über Differentialgleichungen. J. Reine Angew. Math. 144, 284-313 (1914) (in German)

8. Massera, J.L., Schaeffer, J.J.: Linear differential equations and functional analysis. Ann. Math. 67, 517-573 (1958)

9. Massera, J.L., Schaeffer, J.J.: Linear differential equations and functional analysis, iv. Math. Ann. 139, 287-342 (1960)

10. Massera, J.L., Schaeffer, J.J.: Linear Differential Equations and Function Spaces. Academic Press, London (1966)

11. Daleckii, J., Krein, M.G.: Stability of Solutions of Differential Equations in Banach Space. Am. Math. Soc., Providence (1974)

12. Coppel, W.A.: Dichotomies in Stability Theory. Lecture Notes in Mathematics, vol. 629. Springer, Berlin (1978)

13. Barreira, L., Dragičević, D., Valls, C.: From one-sided dichotomies to two-sided dichotomies. Discrete Contin. Dyn. Syst. 35(7), 2817-2844 (2015)

14. Chu, J., Liao, F.-F., Siegmund, S., Xia, Y., Zhang, W.: Nonuniform dichotomy spectrum and reducibility for nonautonomous equations. Bull. Sci. Math. 139(5), 538-557 (2015)

15. Bekryaeva, E.: On the uniformness of estimates for the norms of solutions of exponentially dichotomous systems Differ. Equ. 46, 628-638 (2010)

16. Tien, L.H., Nhien, L.D., Chien, T.V.: Nonuniform exponential dichotomy for block triangular systems on the half line. J. Nonlinear Sci. Appl. 13, 85-96 (2020)

17. Sacker, R.J., Sell, G.R.: A spectral theory for linear differential systems. J. Differ. Equ. 27(3), 320-358 (1978)

18. Siegmund, S.: Dichotomy spectrum for nonautonomous differential equations. J. Dyn. Differ. Equ. 14(1), 243-258 (2002)

19. Barreira, L., Valls, C.: Stability of nonautonomous differential equations in Hilbert spaces. J. Differ. Equ. 217(1), 204-248 (2005)

20. Barreira, L., Vall, C.: Stability in nonautonomous dynamics: a survey of recent results. São Paulo J. Math. Sci. 1(2), 133-173 (2007)

21. Sell, G.R.: The structure of a flow in the vicinity of an almost periodic motion. J. Differ. Equ. 27(3), 359-393 (1978)

22. Barreira, L., Valls, C.: Smooth robustness of parameterized perturbations of exponential dichotomies. J. Differ. Equ. 249(8), 2021-2043 (2010)

23. Wu, H., Li, W.: Poincaré type theorems for non-autonomous systems. J. Differ. Equ. 245(10), 2958-2978 (2008)

24. Pötzsche, C.: Fine structure of the dichotomy spectrum. Integral Equ. Oper. Theory 73(1), 107-151 (2012)

25. Barreira, L., Dragičević, D., Valls, C.: Nonuniform spectrum on the half line and perturbations. Results Math. 72 125-143 (2017)

26. Barreira, L., Dragičević, D., Valls, C.: Nonuniform spectrum on Banach spaces. Adv. Math. 321, 547-591 (2017)

27. Sontag, E.D.: Mathematical Control Theory: Deterministic Finite Dimensional Systems. Springer, New York (2013)

28. Dickinson, B.: On the fundamental theorem of linear state variable feedback. IEEE Trans. Autom. Control 19(5), 577-579 (1974)

29. Makarov, E.K., Popova, S.N.: Controllability of Asymptotic Invariants of Time-Dependent Linear Systems. Belorusskaya Nauka, Minsk (2012)

30. Babiarz, A., Czornik, A., Makarov, E., Niezabitowski, M., Popova, S.: Pole placement theorem for discrete time-varying linear systems. SIAM J. Control Optim. 55(2), 671-692 (2017)

31. Babiarz, A., Banshchikova, I., Czornik, A., Makarov, E., Niezabitowski, M., Popova, S.: Proportional local assignability of Lyapunov spectrum of linear discrete time-varying systems. SIAM J. Control Optim. 57(2), 1355-1377 (2019)

32. Babiarz, A., Banshchikova, I., Czornik, A., Makarov, E.K., Niezabitowski, M., Popova, S.: Necessary and sufficient conditions for assignability of the Lyapunov spectrum of discrete linear time-varying systems. IEEE Trans. Autom. Control 63(11), 3825-3837 (2018)

33. Cuong, L.V., Doan, T.S.: Assignability of dichotomy spectrum for discrete time-varying linear control systems. Discrete Contin. Dyn. Syst., Ser. B 25(9), 3597-3607 (2020)

34. Babiarz, A., Cuong, L.V., Czornik, A., Doan, T.S.: Necessary and sufficient conditions for assignability of dichotomy spectrum of one-sided discrete time-varying linear systems. IEEE Trans. Autom. Control (2021). Early Access. https://doi.org/10.1109/TAC.2021.3073061

35. Babiarz, A., Cuong, L.V., Czornik, A., Doan, T.S.: Necessary and sufficient conditions for assignability of dichotomy spectra of continuous time-varying linear systems. Automatica 125, 109466 (2021)

36. Barreira, L., Valls, C.: Stability theory and Lyapunov regularity. J. Differ. Equ. 232(2), 675-701 (2007)

37. Barreira, L., Dragičević, D., Valls, C.: Tempered exponential dichotomies: admissibility and stability under perturbations. Dyn. Syst. 31(4), 525-545 (2016) 
38. Barreira, L., Rijo, J., Valls, C.: Characterization of tempered exponential dichotomies. J. Korean Math. Soc. 57(1), 171-194 (2020)

39. Battelli, F., Palmer, K.J.: Criteria for exponential dichotomy for triangular systems. J. Math. Anal. Appl. 428(1), 525-543 (2015)

40. Gaishun, I.: Discrete-Time Systems. Natsionalnaya Akademiya Nauk Belarusi. Institut Matematiki, Minsk (2001)

41. Kwakernaak, H., Sivan, R.: Linear Optimal Control Systems, vol. 1. Wiley-Interscience, New York (1972)

42. Agarwal, R.P.: Difference Equations and Inequalities: Theory, Methods, and Applications. CRC Press, New York (2000)

43. Barreira, L., Valls, C.: Tempered exponential behavior for a dynamics in upper triangular form. Electron. J. Qual. Theory Differ. Equ. 2018, 77, 1-22 (2018)

44. Halanay, A., Ionescu, V.: Time-Varying Discrete Linear Systems: Input-Output Operators. Riccati Equations. Disturbance Attenuation. Operator Theory: Advances and Applications, vol. 68. Birkhäuser, Basel (1994)

45. Respondek, W.: Geometric methods in linearization of control systems. Banach Cent. Publ. 1(14), $453-467$ (1985)

46. Jakubczyk, B., Respondek, W.: Feedback equivalence of planar systems and stabilizability. In: Kaashoek, M.A., van Schuppen, J.H., Ran, A.C.M. (eds.) Robust Control of Linear Systems and Nonlinear Control, pp. 447-456. Birkhäuser, Boston (1990)

47. Li, S.-J., Respondek, W.: The geometry, controllability, and flatness property of the n-bar system. Int. J. Control 84(5), 834-850 (2011)

48. Chen, Y., Respondek, W.: Geometric analysis of differential-algebraic equations via linear control theory. SIAM J. Control Optim. 59(1), 103-130 (2021)

49. Horn, R., Johnson, C.: Matrix Analysis. Cambridge University Press, Cambridge (1985)

\section{Submit your manuscript to a SpringerOpen ${ }^{\circ}$} journal and benefit from:

- Convenient online submission

- Rigorous peer review

Open access: articles freely available online

- High visibility within the field

- Retaining the copyright to your article

Submit your next manuscript at $>$ springeropen.com 